This item was submitted to Loughborough's Research Repository by the author.

Items in Figshare are protected by copyright, with all rights reserved, unless otherwise indicated.

\title{
Intra-membrane ligand diffusion and cell shape modulate juxtacrine patterning
}

PLEASE CITE THE PUBLISHED VERSION

LICENCE

CC BY-NC-ND 4.0

\section{REPOSITORY RECORD}

Webb, Steven D., and Markus R. Owen. 2019. "Intra-membrane Ligand Diffusion and Cell Shape Modulate Juxtacrine Patterning". figshare. https://hdl.handle.net/2134/253. 


\title{
Intra-membrane ligand diffusion and cell shape modulate juxtacrine patterning
}

\author{
Steven D WebB and Markus R Owen \\ Department of Mathematical Sciences, Loughborough University, \\ Loughborough, LE11 3TU, UK. \\ E-mail: S.D.Webb@lboro.ac.uk, M.R.Owen@lboro.ac.uk
}

\begin{abstract}
A key problem in developmental biology is how pattern and planar polarity are transmitted in epithelial structures. Examples include Drosophila neuronal differentiation, ommatidia formation in the compound eye, and wing hair polarisation. A key component for the generation of such patterns is direct cell-cell signalling by transmembrane ligands, called juxtacrine signalling. Previous models for this mode of communication have considered homogeneous distributions in the cell membrane, and the role of polarity has been largely ignored. In this paper we determine the role of inhomogeneous protein and receptor distributions in juxtacrine signalling. We explicitly include individual membrane segments, diffusive transport of proteins and receptors between these segments, and production terms with a combination of local and global responses to ligand binding.

Our analysis shows that intra-membrane ligand transport is vital for the generation of long wavelength patterns. Moreover, with no ligand transport, there is no pattern formation for lateral induction, a process in which receptor activation up-regulates ligand production. Biased production of ligand also modulates patterning bifurcations and predicted wavelengths. In addition, biased ligand and receptor trafficking can lead to regular polarity across a lattice, in which each cell has the same orientation - directly analogous to patterns of hairs in the Drosophila wing. We confirm the trends in pattern wavelengths previously observed for patterns with cellular homogeneity - lateral inhibition tends to give short range patterns, while lateral induction can give patterns with much longer wavelengths. Moreover, the original model can be recovered if intra-membrane bound receptor diffusion is included and rapid equilibriation between the sides is considered. Finally, we consider the role of irregular cell shapes and waves in such networks, including wave propagation past clones of non-signalling cells.
\end{abstract}

Key words: Delta-Notch - Juxtacrine - Lateral Inhibition - Morphogenesis - Polarity 


\section{Introduction}

Communication between cells is an essential process during development. Although many intercellular signals function as soluble proteins, certain signals exist as membrane-bound forms and can bind and activate membrane receptors on adjacent cells. This process, called juxtacrine signalling, is vital in various aspects of development and tissue maintenance, being able to deliver intercellular signals while also supporting adhesive interactions between cells (Bosenberg and Massagué, 1993). Some juxtacrine signals exist only in membrane bound forms, e.g. the Drosophila fruit fly proteins Boss and Delta, or are simply precursors of soluble forms, e.g. Transforming growth factor alpha, TGF- $\alpha$ (see Refs (Massagué, 1990; Fagotto and Gumbiner, 1996) for more examples). Both anchored and soluble forms are able to bind and trigger responses in receiving cells, although the relative importance of the paracrine (soluble) and juxtacrine modes in the latter case depends on the cleavage rate of the membrane bound form to the soluble phase. A consequence of these binding interactions is that cells adopt particular programs of gene activation to give spatial patterns of cell fate in the developing embryo.

There have been a number of mathematical models proposed for juxtacrine signalling (Collier et al., 1996; Owen and Sherratt, 1998; Owen et al., 2000). The first was formulated for signalling via the transmembrane ligand Delta binding to its receptor Notch on adjacent cells (Collier et al., 1996). Notch is crucial for developing cells - if this pathway is perturbed, cells fail to differentiate correctly, usually adopting the default neural fate (Bray, 2000a; Lewis, 1996; Lewis, 1998; Whitfield et al., 1997). Delta-Notch is best known for the process called lateral inhibition, whereby high Delta in a cell down-regulates Delta in its neighbours via binding with Notch on their surfaces (Lewis, 1996; Kimble and Simpson, 1997; Haddon et al., 1998). Given sufficiently strong inhibition, this mechanism is capable of generating patterns of Delta/Notch expression with characteristic length scales of two or three cells (Collier et al., 1996). More recent work (Owen and Sherratt, 1998; Owen et al., 2000; Wearing et al., 2000) has shown that patterns with length scales of many cell diameters are generated by the opposite phenomenon of lateral induction, with ligand binding up-regulating production of new ligand and receptor. Such induction is well established for many juxtacrine signals (Reilly and Melton, 1996) including TGF- $\alpha$ and EGF binding to EGF-R (Clark et al., 1985; Coffey et al., 1987) and also the DeltaNotch system in some contexts (De Celis and Bray, 1997; Huppert et al., 1997; Lewis, 1998; Panin et al., 1997). In both these modelling approaches, individual cells are treated as single entities, and it is assumed that all molecules are equally distributed on each 
cell, and that production of new proteins is similarly homogeneous. It is the aim of this paper to describe an extended model that includes inhomogeneous distributions on the cell membrane, and explores the roles of intra-membrane diffusion, biased insertion of new protein, and irregular cell shapes.

The generation of cellular polarity is currently the subject of much experimental research (Bray, 2000b). For example, the eye of the fruit fly, Drosophila, is composed of 800 photoreceptor clusters called ommatidia, each displaying a precise polarity, the ommatidia in the dorsal half pointing in the opposite direction to those in the ventral half (Bray, 2000b; Wehrli and Tomlinson, 1995; Strutt and Mlodzik, 1995; Tomlinson and Struhl, 1999). In the Drosophila wing, each cell orients itself along the proximo-distal axis, generating a hair at the distal vertex (Eaton, 1997). In both cases, this planar polarity requires the transmembrane receptor Frizzled (Weber et al., 2000; Bray, 2000b; Tomlinson and Struhl, 1999), and an as yet unidentified Factor X (Bray, 2000b), that carries the polarizing signal between cells. The secreted protein Four-jointed may be part of the Factor X signal (Bray, 2000b; Zeidler et al., 1999), and the protein Flamingo may also play an important role (Tomlinson and Struhl, 1999; Usui et al., 1999), but it is not clear whether polarity is transmitted via gradients of these proteins across each cell or between cells. Models must allow for inhomogeneous distributions of ligand and receptor on the surface of individual cells to distinguish between these two possibilities. Before we describe the development and analysis of a segmental model, we will present a summary of the previous results.

\subsection{Previous work}

The model of Collier et al (Collier et al., 1996) was formulated in terms of activity of a protein and its receptor, incorporating a negative feedback loop in which receptor activation down-regulates ligand production. More detailed models reflect the elementary binding events of signalling molecules binding to free receptors (Owen and Sherratt, 1998; Owen et al., 2000). This Ligand-Receptor model, henceforth referred to as the L-R model, consists of ODEs for the numbers of ligand molecules, unoccupied receptors and occupied receptors, on the surface of each cell in a regular two dimensional epithelial sheet. The kinetics are based on a generic scheme similar to that of Waters et al (Waters et al., 1990) for EGF-EGF-R interactions: ligand on the surface of one cell binds reversibly to free receptors on the surface of immediately neighbouring cells. The resulting bound receptors stimulate activation or inhibition of ligand and receptor production, characterised 
by the slopes, $\mathcal{A}$ and $\mathcal{F}$, of the two production functions. In this way, activation (inhibition) of ligand production corresponds to $\mathcal{A}>0(\mathcal{A}<0)$, and activation (inhibition) of receptor production corresponds to $\mathcal{F}>0(\mathcal{F}<0)$. Surprisingly, lateral induction of ligand production $(\mathcal{A}>0)$ has been shown to generate long wavelength patterns (Owen and Sherratt, 1998; Owen et al., 2000; Wearing et al., 2000), contrary to the prevailing wisdom that induction should lead to homogeneity (Lewis, 1998). Note that wavelength refers to the number of cells between peaks in a regular pattern of bound receptor expression.

More recent work on a variety of geometries (strings, squares and hexagons) shows that longer wavelengths are still predicted even with lateral inhibition of ligand production $(\mathcal{A}>0)$, provided that there is sufficient receptor upregulation (Webb and Owen, 2003). This runs counter to current thinking in developmental biology, and contrasts with previous predictions that only very short wavelength patterns are possible (Collier et al., 1996). In fact, a connection can be made with the activity-based models of Collier et al (Collier et al., 1996) when there is a constant level of receptor expression and slow binding and dissociation - this limit allows for an equivalence of the patterned steady states, and bifurcations, of both models. When ligand interactions are not slow, the reduction cannot be made, and the role of the cellular geometry is crucial. In particular, patterns in hexagonal arrays with lateral inhibition of ligand requires significant activation of receptor production, or unrealistically strong ligand inhibition.

Figure 1 illustrates the region of parameter space that gives a patterning instability in square and hexagonal arrays. By patterning instability we mean that a homogeneous steady state is both stable to homogeneous perturbations and unstable to inhomogeneous perturbations. This instability leads to pattern formation in the shaded regions: black indicates instability to wavelength 2 cell patterns ( 3 in hexagons), lighter shading is for longer wavelengths. For all geometries, wavelengths increase from left to right, and the longest wavelengths are predicted for strongest activation of ligand production and the weakest for receptor production in the $\mathcal{A}, \mathcal{F}>0$ pattern region. Other novel behaviours include a Hopf bifurcation to temporally oscillating patterns, taking the form of either standing or travelling waves (Webb and Owen, 2003). This observation is particularly relevant given the recent interest in the cellular mechanisms involved in somitogenesis.

The pattern region is delimited by four straight lines $\left(T_{1}, H_{1}, T_{\kappa}\right.$ and $H_{\kappa}$, where $\kappa=-1$ for strings or squares, and $\kappa=-1 / 2$ for hexagons) and an ellipse $\left(T_{d}\right)$ that intersects two of those lines tangentially (as seen in Boxes 1 and 2). It appears in Figure 1(b) that the pattern region for hexagons is bounded by a horizontal line $T_{-1 / 2}$, but in fact the line has 
a very shallow positive slope in comparison to the line $T_{-1}$ for squares. This means that it is harder to get patterns in hexagons with lateral inhibition when receptor activation is weak or production is inhibited. These lines and curves are derived in (Webb and Owen, 2003), and will be referred to later. Figure 2 shows the results of simulations for different strengths of ligand and receptor production, corresponding to different locations in this $(\mathcal{A}, \mathcal{F})$ pattern region. The first two columns are for lateral inhibition $(\mathcal{A}<0$, strongest inhibition on the left), and the third column is for lateral induction $(\mathcal{A}>0)$. In each case receptor production is up-regulated $(\mathcal{F}>0)$. As we see, decreasing lateral inhibition gives longer wavelengths, and for lateral induction the wavelengths are longer still, for all geometries.

Building on this previous work, in the following we extend the L-R model for juxtacrine signalling to include inhomogeneous distributions on the cell membrane by modelling individual membrane segments, rather than whole cells. In section 2 we develop the model. Section 3 describes a detailed analysis of patterning in strings of cells, and outlines the features seen for arrays of square and hexagonal cells. Section 4 elucidates the effect of intra-membrane ligand and receptor diffusion, and the role of polarised trafficking of new ligand and/or receptors. In section 5 we descibe a number of simulations, including the role of irregular cellular polarity, clones of defective cells, and irregular geometries. We conclude with a discussion in section 6 .

\section{Model development}

The development of a segmental model rests upon two key extensions of the L-R model. The first is to relax the assumption of equal distributions on the cell surface, and allow different sides of a cell to have different numbers of ligand and receptor molecules. This requires a model which keeps track of each membrane surface separately. The second extension is that production may also be localised to a particular side of a cell. Potential mechanisms for such localisation include local protein synthesis, active transport from intracellular stores, and selective degradation (Strutt, 2002). Thus production of new ligand or receptor may be regarded as central, in response to the total number of bound receptors on all sides, and with distribution to each side according to its proportion of the total cell surface; or as local, with production in response to the number of receptors at a particular side directed only to that side. We restrict attention to the formation of patterns in strings of cells, and square and hexagonal arrays. The labelling schemes 
for these geometries are illustrated in Figure 3. Note that for simplicity we neglect any variation in the depth of the string or array of cells.

To extend the original model for juxtacrine signalling to include inhomogeneous distributions on the cell membrane, we divide the membrane of each cell into a finite number of segments, and interactions between cells only occur on adjacent sides of neighbouring cells. Our model variables are concentrations of ligand, $a(\mathbf{x}, t)$, receptors, $f(\mathbf{x}, t)$, and bound receptors, $b(\mathbf{x}, t)$, at spatial position $\mathbf{x}$ and time $t$, with units number of molecules per cell segment. This is equivalent to number per unit area of cell membrane where each cell consists of identical sides. The model equations are

$$
\begin{aligned}
& \frac{d a}{d t}=\underbrace{-k_{a} a \hat{f}}_{-k_{a} \hat{a} f}+\overbrace{k_{d} b}^{\text {binding }}-\overbrace{d_{f} f}^{k_{d} \hat{b}}-\overbrace{P_{f}(b,\langle b\rangle)}^{\text {dissociation }}+\overbrace{D_{f} \Delta(f)}^{d_{a} a}+\underbrace{\text { decay }}_{\overbrace{a} P_{a}(b,\langle b\rangle)}+\underbrace{\text { transport }}_{\underbrace{}_{a} D_{a} \Delta(a)} \\
& \frac{d f}{d t}=\overbrace{k_{a} \hat{a} f}^{\text {binding }}-\overbrace{k_{d} b}^{\text {dissociation }}-\overbrace{k_{i} b,}^{\text {internalisation }}
\end{aligned}
$$

where

$$
\mathcal{A}=\check{P}_{a}^{\prime}\left(N b_{e}\right), \quad \mathcal{F}=\check{P}_{f}^{\prime}\left(N b_{e}\right), \quad \text { and } \quad \check{P}_{u}\left(N b_{e}\right)=N\left(d_{u} u_{e}+k_{i} b_{e}\right) \quad \text { for } u=a, f,
$$

and the parameters $k_{a}, k_{d}, k_{i}, d_{a}, d_{f}, D_{a}$ and $D_{f}$ are all real scalars. Hats indicate the number on the adjacent membrane segment of a neighbouring cell. The first three terms of each equation represent ligand-receptor binding between adjacent segments, linear decay, and bound receptor internalisation. For example, the term $-k_{a} a \hat{f}$ represents the juxtacrine binding of free ligands to neighbouring free receptors, according to the law of mass action with rate constant $k_{a}$. Diffusive transport of proteins between segments of the same cell is included, $\Delta(u)$ where $u$ is either $a$ or $f$. Since we will consider a finite number of segments, $\Delta(u)$ will be a discretised approximation. Thus, $\Delta\left(u_{i}\right)=u_{i+1}-2 u_{i}+u_{i-1}$, where $u_{i}$ is the quantity of $u$ on side $i$ and $i \pm 1$ is calculated modulo the number of sides on that cell.

We consider production terms, $P_{u}(b,\langle b\rangle), u=a, f$, with a combination of local and global responses to the numbers of bound receptors on the segment, $b$, and the total number of bound receptors on the whole cell, $\langle b\rangle=\sum_{i=1}^{N} b_{i}$ :

$$
P_{u}(b,\langle b\rangle)=\frac{\check{P}_{u}(\langle b\rangle)}{N}\left(1-\gamma_{u} \frac{(\langle b\rangle-N b)}{\langle b\rangle}\right),
$$


where $\check{P}_{u}(\langle b\rangle)$ is the total production for the cell, $\gamma_{u}$ is a parameter used to vary the relative contribution of the local and global responses and $N$ is the number of sides. For $\gamma_{u}=0$, production is purely global, but when $\gamma_{u} \neq 0$, each side receives a weighted proportion of the total production for the cell. When $\gamma_{u}>0$, the bias is toward the sides with the most bound receptors. If $\gamma_{u}<0$, the opposite is true, and the side with the least bound receptors receives the largest proportion of the total production. The particular forms we take for $P_{a}$ and $P_{f}$ are described in Appendix D.

We use linear analysis similar to that by Turing (Turing, 1952) to investigate patterning instabilities in this system. This analysis is similar to that used previously (Webb and Owen, 2003), but for completeness we include the details in Appendices A and B.

\section{Analysis of Pattern Formation}

Linearisation and substitution of perturbations of the form $u=\bar{u} \exp (\alpha t+i \lambda \cdot \mathbf{x})$ gives a characteristic equation, $P(\alpha, \lambda)$, whose roots determine the growth rate $\alpha$ for the wavenumber $\lambda$. Here, $u=a, f, b$, and for a string of cells $\lambda=2 \pi / \omega$; for squares and hexagons $\lambda=\left(\lambda_{1}, \lambda_{2}\right)$, where $\lambda_{1}=2 \pi / \omega_{1}$ and $\lambda_{2}=2 \pi / \omega_{2}$ are the wave lengths for the two directions $j$ and $l$. In Appendix A we consider patterning bifurcations in detail for a string of cells, and demonstrate similar features for square and hexagonal arrays.

\subsection{String of cells}

We first consider where the homogeneous equilibrium is stable in the absence of any spatial effects. In Appendix A.1 we show that two lines in the $(\mathcal{A}, \mathcal{F})$ parameter space, labelled $T_{1}$ and $H_{1}$, delimit the region of stability - see equation (13). Both $T_{1}$ and $H_{1}$ have negative slope and intersect the axis at a positive value of $\mathcal{F}$. The homogeneous steady state is stable if $\mathcal{F}$ lies below both of these lines. We remark that these lines are exactly the same as those found for homogeneous stability in the original L-R model (Webb and Owen, 2003). 


\section{Instability to inhomogeneous perturbations}

We now consider where the steady state is unstable to patterned perturbations. First, we consider the case when the bifurcation arises for a period two pattern of alternating high and low cell fates. Conveniently, the patterning bifurcations occur on the lines $T_{-1}$ and $H_{-1}$ (equation (15)) and the equilibrium is unstable to inhomogeneous perturbations when $\mathcal{F}>\min \left\{T_{-1}, H_{-1}\right\}$. Note that the same lines appear in the original model, and that these lines are precisely the reflection in the $\mathcal{F}$ axis of the lines $T_{1}$ and $H_{1}$ that govern homogeneous stability.

Other bifurcations are possible (i.e. for wavelengths other than two). We show in Appendix A.1 that a patterning bifurcation occurs along an ellipse $T_{d}$ in $\mathcal{A}-\mathcal{F}$ space that intersects the two lines $T_{ \pm 1}$ tangentially. It turns out that this bifurcation, together with the lines $H_{ \pm 1}$, appears to give all the biologically relevant conditions for pattern formation in the $\mathcal{A}-\mathcal{F}$ plane. The points at which the two lines $T_{ \pm 1}$ touch the curve $T_{d}$ are, respectively $\left(\mathcal{A}_{\mathrm{p}}, T_{1}\left(\mathcal{A}_{\mathrm{p}}\right)\right)$ and $\left(-\mathcal{A}_{\mathrm{p}}, T_{-1}\left(-\mathcal{A}_{\mathrm{p}}\right)\right)$. See Figure 4 for a qualitative illustration of these curves in the $(\mathcal{A}, \mathcal{F})$ plane.

So far, we have seen that the pattern region in $\mathcal{A}-\mathcal{F}$ space is bounded by four straight lines $\left(T_{1}, H_{1}\right.$ for stability to homogeneous perturbations and $T_{-1}, H_{-1}$ for instability to inhomogeneous perturbations) and an ellipse $T_{d}$ - which is the same configuration of lines and curves as in the original model, except that the ellipse has a slightly different form (Webb and Owen, 2003). Crossing the line $H_{-1}$ corresponds to a Hopf bifurcation, but we have been unable analytically to identify other possible Hopf bifurcations. However, we expect similar behaviour as in the original model in which the other bifurcations are only possible for very strong (positive and negative) receptor feedback - stronger than can be expected biologically for our parameter set. We therefore expect that these bifurcations are not significant in the sides model, and that, for realistic parameter values, patterns are only predicted in the region defined by the lines $T_{ \pm 1}, H_{ \pm 1}$ and curve $T_{d}$. This is supported by numerical calculations of the unstable modes - the results are illustrated in Figure 5(a): patterns are predicted in the shaded region, areas that are not shaded are either unstable to homogeneous perturbations or stable to inhomogeneous perturbations.

In summary, the homogeneous steady state is stable to homogeneous perturbations when $\mathcal{F}<T_{1}$ and $\mathcal{F}<H_{1}$. Within this region, pattern formation is predicted for $\mathcal{F}>$ $\min \left\{T_{-1}, H_{-1}, T_{d}\right\}$. There are four possible geometries for this region, according to the relative slopes of the lines $T_{-1}$ and $H_{-1}$, and the location of their point of intersection. 
For $d_{f}>d_{a}$, the line $T_{-1}$ is steeper than $H_{-1}$, and they intersect for $\mathcal{F}>0$, with $\mathcal{A}$ taking either sign. For $d_{f}<d_{a}$, the line $H_{-1}$ is steeper than $T_{-1}$, and these two lines intersect for $\mathcal{F}<T_{\kappa}$ and $\mathcal{A}<0$. Thus, as in the original model, there is a region of $\mathcal{A}$ and $\mathcal{F}$ space in which the steady state is unstable to patterns via a Hopf bifurcation, and limit cycle oscillations are predicted; this is when $\mathcal{F}<T_{-1}$ and $\mathcal{F}>H_{-1}$. Numerical investigation shows that these oscillations take the form of either a standing wave, in which each cell oscillates out of phase with its neighbours in a period 2 pattern, or a travelling wave (illustrated in Figure 6 for a string of cells). Note that as the wave propagates it causes an alternating polarisation of activation between the two sides of a cell. We remark that it is also possible to cross directly through a Hopf for $\mathcal{F}>0$ via the line $H_{-1}$ (see Figure 4 ).

\subsection{Square and Hexagonal arrays}

The labelling schemes for square and hexagonal arrays are illustrated in Figure 3(b) and (c), respectively. It is straightforward to calculate the stability matrix for these systems. However, due to the scale of this problem - a $12 \times 12$ matrix for squares and $18 \times 18$ for hexagons - we are restricted to the numerical calculation of the eigenvalues to determine the stability of the homogeneous steady state in the $(\mathcal{A}, \mathcal{F})$ plane. Figure $5(\mathrm{~b})$ and $(\mathrm{c})$ shows the results of these calculations for the two geometries - pattern formation is predicted in the shaded regions. In general, the pattern region for squares/hexagons is qualitatively similar to that for strings — being bounded by four straight lines and a curve that sits tangentially between two these lines - except that the corresponding curve $T_{d}$ for squares sits just below that for strings in $\mathcal{A}-\mathcal{F}$ space, and the line delimiting the lower part of the pattern region for hexagons has a much shallower slope. It appears in Figure 5(b) that the pattern region for squares is bounded by the same four straight lines, $T_{ \pm 1}$ and $H_{ \pm 1}$, as strings. In Appendix A.2, we show that this is indeed the case.

As in the original model, the pattern region for hexagons is smaller than that for strings/squares (see Figure 5(c)). This arises because the line delimiting the lower part of the pattern region for hexagons has a much shallower slope compared to that for strings/squares. Obtaining an expression for this line for hexagons is difficult. However, it appears that the upper boundaries are the same for all geometries - given by $T_{1}$ and $H_{1}$.

As in strings, spatiotemporal oscillations are predicted for squares in the region bounded by the lines $T_{-1}$ and $H_{-1}$ when $d_{f}<d_{a}$. Characterising these behaviours analytically for hexagons is difficult because we cannot solve explicitly for the corresponding lines. How- 
ever, numerical investigation of the $(\mathcal{A}, \mathcal{F})$ parameter space shows that such an oscillatory regime does indeed exist for hexagons when there is weak or negative receptor feedback and very strong negative ligand feedback.

\section{Intra-membrane protein diffusion and polarity}

With no intra-membrane ligand diffusion, $D_{a}=0$, the ellipse $T_{d}$ is not defined. In this case, the pattern region is smaller, being the region in $(\mathcal{A}, \mathcal{F})$ space bounded by the four straight lines $T_{ \pm 1}, H_{ \pm 1}$. In particular there is no pattern formation for activation of ligand production $(\mathcal{A}>0)$. Figure 7 shows how increasing $D_{a}$ increases the size of the pattern region and makes pattern formation with lateral induction possible. As $D_{a}$ increases, the ellipse $T_{d}$ becomes flatter, and the tangential intersections with the lines $T_{ \pm 1}$ occur for smaller $\mathcal{F}$, making the patterning region larger. In addition, as $D_{f}$ increases, the centre and bottom of the ellipse are shifted slightly in the positive $\mathcal{F}$ direction. For further details see Appendix B.

We remark that the original model may be recovered if intra-membrane bound receptor diffusion is also included with the coefficient $D_{b}$, and the limit $D_{a}, D_{f}, D_{b} \rightarrow \infty$ is considered. It is important to identify this requirement, even though it seems highly unlikely given that bound receptors are connected to two cells via ligand. However, even for rapid equilibriation of ligand and free receptor between the sides $\left(D_{a}, D_{f} \rightarrow \infty\right)$ we still obtain very similar expressions to the original model (see Table 1). These show that the top of the ellipse is basically the same, but the centre and bottom are raised slightly up the $\mathcal{F}$-axis in the sides model.

Our calculations show that the same trends are observed for patterns in squares and hexagons when intra-membrane ligand diffusion, $D_{a}$, is varied: when $D_{a}=0$, there is no pattern formation for $\mathcal{A}>0$; increasing $D_{a}$ enlarges the pattern region about $\mathcal{A}=0$ and makes patterns with $\mathcal{A}>0$ possible. In addition, decreasing $D_{a}$ makes it easier to get patterns for hexagons via lateral inhibition $(\mathcal{A}<0)$, with negative receptor feedback $(\mathcal{F}<0)$, by making the lower part of the pattern region steeper - see Figure 8 . The thin diagonal lines indicate the bifurcations; the solid line is when $D_{a}=0$, and the arrows indicate the diection of increasing $D_{a}$. In the limit $D_{a}, D_{f}, D_{b} \rightarrow \infty$, this lower boundary coincides exactly to that derived for hexagons in the original L-R model (Webb and Owen, 2003) (line $T_{-1 / 2}$ in Figure $1(\mathrm{~b})$ ). 
We now consider how altering the bias in ligand and receptor production, $\gamma_{a}, \gamma_{f} \neq 0$, affects patterning. For the case of biased receptor production, $\gamma_{f} \neq 0$, we only observe that the centre and bottom of the ellipse are shifted slightly along the $\mathcal{F}$-axis. However, when biased ligand production is included, $\gamma_{a} \neq 0$, the ellipse loses its symmetry about the $\mathcal{F}$-axis. Figure 9 shows that biased ligand production modulates the patterning region by tilting the ellipse. Consequently, the pattern region for $\mathcal{A}>0$ becomes smaller when the bias is towards the side with the most bound receptors $\left(\gamma_{a}>0\right)$, and larger for an opposite bias in ligand production $\left(\gamma_{a}<0\right)$ (compare dashed and solid ellipse in Figure 9 - dashed ellipses show how the patterning bifurcations move when $\gamma_{a}= \pm 1$ ).

\subsection{Pattern wavelengths}

Intra-membrane transport and biased ligand production also modulate the patterning wavelengths. The effects are illustrated in Figure 9 for a string of cells. The fastest growing modes are found via numerical calculation of the wavelengths, $\omega$, which give the largest real parts for the roots of the dispersion relation $P(\alpha)$. They are illustrated on a grey scale, in which black indicates wavelength 2 cell patterns and lighter shading indicates longer wavelengths. The solid diagonal lines and ellipses are the analytically derived bifurcations, and the rays emanating from the ellipses indicate the transitions of the fastest growing mode when $\gamma_{a}=0$. The dashed ellipses show the bifurcations when $\gamma_{a}= \pm 1$, and the shadings indicate the transitions between fastest growing modes. In parts (a) and (b) $\gamma_{a}=-1$, in (c) and (d) $\gamma_{a}=1$. As the figure demonstrates, increasing $D_{a}$ enlarges the patterning region but it also makes longer wavelengths more likely (compare left and right columns). With no intra-membrane ligand diffusion, $D_{a}=0$, only short wavelengths are predicted. Altering the bias in ligand production also modulates patterning wavelength (compare upper and lower rows). We see that enhancing ligand production on the side with the most bound receptors $\left(\gamma_{a}>0\right)$ shortens predicted wavelengths, whereas an opposite bias for ligand increases patterning wavelengths. The pictures for squares and hexagons are similar - Figure 5(b) and (c) shows the variations of fastest growing modes in $(\mathcal{A}, \mathcal{F})$ space for these geometries in the case of unbiased global feedback and when intra-membrane ligand diffusion is $D_{a}=0.1$.

Figure 10(a)-(c) illustrates the effects of different combinations of these biased productions in strings of cells. Each simulation is for the same strengths of lateral inhibition and positive receptor production. In each case the boundary conditions are periodic, and the initial conditions are a small perturbation to the left-hand side of the first cell. We 
see that even with unbiased global production there are still differences between sides due to ligand-receptor binding (part (a)). Enhancing ligand production on the side with the most bound receptors $\left(\gamma_{a}>0\right)$ increases differences between sides and shortens predicted wavelengths (part (b)). In addition, opposite biases (part (c)) increase pattern wavelengths, as predicted.

We can also generate a regular pattern of polarity across the lattice, in which each cell has the same orientation. To generate these patterns in a string (Figure 10(d)), we simply need $\gamma_{a}<0$ and $\gamma_{f}>0$ and parameters within the region of stability in the $(\mathcal{A}, \mathcal{F})$ plane. This leads to greater receptor production on the side with the most bound receptors, allowing still greater increases in bound receptor numbers, and stimulates ligand production on the adjacent side of the neighbouring cell. Simulations indicate that opposite biases, as defined in terms of (3), do not have the same effect in two-dimensions. A simple mechanism that can establish regular two-dimensional polarity is to compare opposite sides of a single cell, rather than compare each side to the average across the whole cell:

$$
P_{u}^{i}(b,\langle b\rangle)=\frac{\check{P}_{u}(\langle b\rangle)}{N}\left(1+\gamma_{u} \frac{b_{i}-b_{j}}{b_{i}+b_{j}}\right), \quad \text { where } j=\left(i+\frac{N}{2}\right) \bmod N .
$$

Here, $P_{u}^{i}$ indicates the part of production that is directed to the $i$ th side. With this form, choosing $\gamma_{a}=-1$ and $\gamma_{f}=1$ achieves the desired effect. In Figure 11, we have explored how defects in signalling in a small group of cells can propagate into the surrounding region of square cells that have regular polarity. Such defects have a direct experimental analogue, where they result in a small number of characteristic behaviours, such as cruciform or spiral arrangements.

\section{Simulations in regular and irregular 2-dimensional arrays}

In two dimensions, the predicted feature of increasing wavelengths with $\mathcal{A}$ is observed, and we can also examine the effect of irregular arrays. In Figure 12, we simulate (1) on hexagonal arrays with varying random perturbations of a regular structure - Appendix E includes details of the extension of (1) to cater for such geometries. Relatively small structural perturbations allow the maintenance of regular patterns, but larger perturbations lead to some irregularities.

As illustrated in (Owen, 2002), the L-R model with homogeneous cells supports travelling 
waves connecting inactive and active homogeneous steady states. When the inactive state is unstable to inhomogeneous perturbations, wave speeds are governed by linearisation ahead of the wave, and small perturbations ahead of the wave (such as would be caused by stochastic effects) become amplified so that the wave is lost. When the inactive state is stable, the wave speed is governed by nonlinearities, as in the bistable reaction diffusion equation. Importantly, small perturbations ahead of the wave decay. When the active state is unstable to spatial perturbations, this provides a robust mechanism for laying down a regular pattern, since random pertubations of the active state tend to lead to irregular states, even though there is a characteristic length scale. Figure 13 shows three simulations illustrating these features of patterning and wave propagation in the extended L-R model with inhomogeneous protein distributions and irregular geometries. The first row shows the evolution from a random perturbation of the active homogeneous steady state. An irregular pattern develops, but it has a characteristic length scale. The second row illustrates the propagation of a wave connecting stable homogeneous steady states

- behind the wave front a regular two dimensional pattern evolves. The third row illustrates how juxtacrine waves can propagate around clones of non-signalling cells. This is in contrast to suggestions that juxtacrine relay mechanisms may be ruled out by such experiments (Strutt, 2002).

\section{Discussion}

Many patterns in early development have a very small spatial scale, and there is much evidence that a crucial role is played by juxtacrine signalling, whereby cells signal directly to their neighbours via membrane-bound proteins (Fagotto and Gumbiner, 1996). Previous models for juxtacrine signalling have considered cells as single entities, and assumed homogeneous distributions of proteins and receptors on the cell membrane. However, inhomogeneous distributions are common, and epithelial structures are often oriented with a certain polarity: for example, cuticular structures in insect epithelia such as hairs, bristles or ridges all orient in the same direction (usually posteriorly or distally) (Wehrli and Tomlinson, 1995; Eaton, 1997). In addition, production of new transmembrane proteins may also be inhomogeneous. Building on previous work (Owen and Sherratt, 1998; Wearing et al., 2000; Webb and Owen, 2003), in this paper we have determined the role of inhomogeneous ligand and receptor distributions in juxtacrine signalling.

The specific mathematical model that we consider was originally developed in (Owen 
and Sherratt, 1998), and provides a generic representation for juxtacrine signalling in an epithelial sheet. This model assumes cellular homogeneity, with model variables being the numbers of ligand molecules, free receptors, and bound receptor-ligand complexes on the surfaces of cells in fixed cellular arrays. To include inhomogeneous distributions on the cell surface, we divided the membrane into a finite number of segments, and tracked protein numbers on each segment. We have considered production terms with a combination of responses to local and global measures of bound receptors, and we also allow linear diffusive transport between segments of the same cell. We find that intra-membrane ligand diffusion is critical for the generation of long wavelength patterns. Moreover, with no ligand transport, there is no pattern formation for lateral induction. Increasing intramembrane ligand diffusion enlarges the patterning region and makes longer wavelengths more likely. It is important to verify that this occurs for relevant parameter ranges. For example, significant enlargement of the pattern region due to transmembrane diffusion is seen for $D_{a}=0.01$ (see Figure 8), which is well within the range for biologically relevant parameter values (see Appendix D). The original model (Owen and Sherratt, 1998) may be recovered if intra-membrane bound receptor diffusion is also included, and the limit of instantaneous equilibriation of ligand, free and bound receptors between the sides is considered. However, this kind of limiting behaviour seems highly unlikely given that bound receptors are connected to two cell membranes via ligand binding.

When ligand transport is included, our analysis confirms the trends in pattern wavelength previously observed for patterns when protein distributions are homogeneous on a single cell - lateral inhibition with weak receptor upregulation gives short range patterns, these give way to longer patterns as receptor production is increased, and lateral induction can give patterns with much longer wavelengths. We have also explored the effects of different combinations of biased ligand and receptor production. Biased production of ligand modulates the patterning region, although this has less effect than varying intramembrane ligand diffusion. Enhancing ligand production on the side with the most bound receptors enhances differences between sides and shortens predicted wavelengths, whereas an opposite bias in ligand production has a homogenising effect and increases pattern wavelengths. We find that even unbiased global production still gives differences between sides due to ligand-receptor binding. In addition, opposite bias for ligand and receptor can lead to the more recognised type of polarity, in which a field of cells have the same orientation. Such patterns have a direct analogue to the coherent arrays of defined polarity in bristles and hairs in Drosophila (Eaton, 1997). However, simple opposite biases in two dimensional arrays of cells do not lead to regular polarity. Rather, we must compare pairs 
of opposite sides, instead of comparing a side with the average binding on a whole cell. More complex mechanisms that may lead to such behaviour include diffusible intracellular modulators that activate or inhibit protein insertion at distant membrane locations. It is also possible that a Turing instability can lead to a polarized pattern on a single cell, and this behaviour has indeed been observed in a continuous activator-inhibitor model for Min proteins in E.Coli (Meinhardt and de Boer, 2001). However, it is important to note that, without such a diffusion driven instability, regular polarity requires biased protein trafficking (see Appendix C).

We have also investigated the role of waves in the initiation of regular patterns. Random perturbations of the active homogeneous steady state lead to an irregular pattern with a characteristic length scale. In contrast, pattern initiation by a wave of activation can lead to more regular structures - in particular, bistability makes this process robust to perturbations ahead of the wave front. An important prediction is that juxtacrine waves can propagate around clones of non-signalling cells, contrary to suggestions that such clones should lead to long range disruption of relay induced patterning (Strutt, 2002).

We found in (Webb and Owen, 2003) that cellular geometry is crucial for patterning, and similar trends are also observed here. In particular, pattern formation via lateral inhibition in hexagonal arrays requires significant activation of receptor production, or unrealistically strong lateral inhibition - however, these patterns are made more likely when there is very little or no intra-membrane ligand diffusion (see Figure 8). Patterning for irregular cell shapes with varying numbers of sides may be constrained by similar restrictions. Appendix E extends the segmental model (1) to a general framework for irregular geometries. In this paper we have explored the effect of perturbing regular cell shapes, but retaining a fixed number of sides. Future work will relax this assumption, and will determine the effect of cell side number and irregularity on patterning bifurcations. Further natural extensions are the inclusion of cell division, death and cell movement. Important mathematical questions also arise when temporal delays due to gene transcription are included, and a combination of paracrine and juxtacrine signalling will lead to a detailed understanding of cellular signalling and applications to specific developmental situations. 


\section{Acknowledgements}

SDW was supported by grant no. GR/R46366/01 from the Engineering and Physical Sciences Research Council of Great Britain.

\section{References}

Bosenberg, M. W. and Massagué, J. (1993). Juxtacrine cell signalling molecules. Curr. Opin. Cell Biol., 5:832-838.

Bray, S. (2000a). Notch. Current Biology, 10:R433-R435.

Bray, S. (2000b). Planar polarity: Out of joint? Current Biology, 10:R155-R158.

Clark, A. J. L., Ishii, S., Richert, N., Merlino, G. T., and Pastan, I. (1985). Epidermal growth factor regulates the expression of its own receptor. Proc. Nat. Acad. Sci. USA, 82:8374-8378.

Coffey, R. J., Derynck, R., Wilcox, J. N., Bringman, T. S., Goustin, A. S., Moses, H. L., and Pittelkow, M. R. (1987). Production and auto-induction of transforming growth Factor- $\alpha$ in human keratinocytes. Nature, 328:817-820.

Collier, J. R., Monk, N. A. M., Maini, P. K., and Lewis, J. H. (1996). Pattern formation by lateral inhibition with feedback: A mathematical model of delta-notch intercellular signalling. J. Theor. Biol., 183:429-446.

De Celis, J. F. and Bray, S. (1997). Feedback mechanisms affecting notch activation at the dorsoventral boundary in the Drosophila wing. Development, 124:3241-3251.

Eaton, S. (1997). Planar polarity in Drosophila and vertebrate epithelia. Curr. Opin. Cell Biol., 9:860-866.

Fagotto, F. and Gumbiner, B. M. (1996). Cell contact dependent signalling. Dev. Biol., 180:445-454.

Haddon, C., Smithers, L., Schneider-Maunoury, S., Coche, T., Henrique, D., and Lewis, J. (1998). Multiple delta genes and lateral inhibition in zebrafish primary neurogenesis. Development, 125:359-370. 
Huppert, S. S., Jacobson, T. L., and Muskavitch, M. A. T. (1997). Feedback regulation is central to Delta-Notch signalling required for Drosophila wing vein morphogenesis. Development, 124:3283-3291.

Kimble, J. and Simpson, P. (1997). The LIN-12/Notch signalling pathway and its regulation. Ann. Rev. Cell Dev. Biol., 13:333-361.

Lauffenburger, D. A. and Linderman, J. (1996). Receptors: Models for Binding, Trafficking and Signalling. Oxford University Press.

Lewis, J. (1996). Neurogenic genes and vertebrate neurogenesis. Curr. Op. Neurobiol., $6: 3-10$.

Lewis, J. (1998). Notch signalling and the control of cell fate choices in vertebrates. Semin. Cell Dev. Biol., 9:583-589.

Massagué, J. (1990). Transforming growth Factor- $\alpha$ : A model for membrane-anchored growth factors. J. Biol. Chem., 265:21393-21396.

Meinhardt, H. and de Boer, P. A. J. (2001). Pattern formation in escherichia coli: A model for the pole-to-pole oscillations of Min proteins and the localization of the division site. Proc. Nat. Acad. Sci. USA, 98:14202-14207.

Owen, M. R. (2002). Waves and propagation failure in discrete space models with nonlinear coupling and feedback. Physica D, 173:59-76.

Owen, M. R. and Sherratt, J. A. (1998). Mathematical modelling of juxtacrine cell signalling. Math. Biosci., 153:125-150.

Owen, M. R., Sherratt, J. A., and Wearing, H. J. (2000). Lateral induction by juxtacrine signalling is a new mechanism for pattern formation. Dev. Biol., 217:54-61.

Panin, V. M., Papayannopoulos, V., Wilson, R., and Irvine, K. D. (1997). Fringe modulates Notch-ligand interactions. Nature, 387:908-912.

Reilly, K. M. and Melton, D. A. (1996). Short-range signalling by candidate morphogens of the TGF beta family and evidence for a relay mechanism of induction. Cell, $86: 743-754$.

Strutt, D. I. (2002). The asymmetric subcellular localisation of components of the planar polarity pathway. Sem. Cell Dev. Biol., 13:225-231. 
Strutt, D. I. and Mlodzik, M. (1995). Ommatidial polarity in the drosophila eye is determined by the direction of furrow progression and local interactions. Development, 121:4247-4256.

Tomlinson, A. and Struhl, G. (1999). Decoding vectorial information from a gradient: Sequential roles of the receptors frizzled and notch in establishing planar polarity in the drosophila eye. Development, 126(24):5725-5738.

Turing, A. M. (1952). The chemical basis of morphogenesis. Phil. Trans. R. Soc. Lond. $B, 237: 37-72$.

Usui, T., Shima, Y., Shimada, Y., Hirano, S., Burgess, R. W., Schwarz, T. L., Takeichi, M., and Uemura, T. (1999). Flamingo, a seven-pass transmembrane cadherin, regulates planar cell polarity under the control of Frizzled. Cell, 98:585-595.

Waters, C. M., Oberg, K. C., Carpenter, G., and Overholser, K. A. (1990). Rate constants for binding, dissociation, and internalization of EGF: Effect of receptor occupancy and ligand concentration. Biochemistry, 29:3563-3569.

Wearing, H. J., Owen, M. R., and Sherratt, J. A. (2000). Mathematical modelling of juxtacrine patterning. Bull. Math. Biol., 62:293-320.

Webb, S. D. and Owen, M. R. (2003). Oscillations and patterns in spatially discrete models for developmental intercellular signalling. J. Math. Biol. To appear.

Weber, U., Paricio, N., and M, M. M. (2000). Jun mediates Frizzled-induced R3/R4 cell fate distinction and planar polarity determination in the Drosophila eye. Development, 127:3619-3629.

Wehrli, M. and Tomlinson, A. (1995). Epithelial planar polarity in the developing drosophila eye. Development, 121(8):2451-2459.

Whitfield, T., Haddon, C., and Lewis, J. (1997). Intercellular signals and cell-fate choices in the developing inner ear: origins of global and of fine-grained pattern. Sem. Cell Dev. Biol., 8:239-247.

Zeidler, M. P., Perrimon, N., and Strutt, D. I. (1999). The four-jointed gene is required in the Drosophila eye for ommatidial polarity specification. Curr. Biol., 9:1363-1372. 


\section{A Analysis of patterning bifurcations}

Linearizing (1) about the homogeneous steady state by setting $a=a_{e}+\tilde{a}, f=f_{e}+\tilde{f}$, and $b=b_{e}+\tilde{b}$, gives (dropping tildes for notational simplicity)

$$
\begin{aligned}
\frac{\partial a}{\partial t} & =-k_{a} a f_{e}-k_{a} a_{e} \hat{f}+k_{d} \hat{b}-d_{a} a \\
& +\frac{1}{N} \mathcal{A}\langle b\rangle+\frac{\gamma_{a} \check{P}_{a}\left(N b_{e}\right)}{N^{2} b_{e}}(N b-\langle b\rangle)+D_{a} \Delta(a) \\
\frac{\partial f}{\partial t} & =-k_{a} \hat{a} f_{e}-k_{a} a_{e} f+k_{d} b-d_{f} f \\
& +\frac{1}{N} \mathcal{F}\langle b\rangle+\frac{\gamma_{f} \check{P}_{f}\left(N b_{e}\right)}{N^{2} b_{e}}(N b-\langle b\rangle)+D_{f} \Delta(f) \\
\frac{\partial b}{\partial t} & =k_{a} \hat{a} f_{e}+k_{a} a_{e} f-k_{d} b-k_{i} b,
\end{aligned}
$$

where

$$
\mathcal{A}=\check{P}_{a}^{\prime}\left(N b_{e}\right), \quad \mathcal{F}=\check{P}_{f}^{\prime}\left(N b_{e}\right), \quad \text { and } \quad \check{P}_{u}\left(N b_{e}\right)=N\left(d_{u} u_{e}+k_{i} b_{e}\right) \quad \text { for } u=a, f .
$$

We will show that the key regulators of model behaviour are the parameters $\mathcal{A}$ and $\mathcal{F}$, which represent the slopes of the feedback functions at the homogeneous steady state.

We look for patterned solutions of the form $a=\bar{a} \exp (\alpha t+i \lambda \cdot \mathbf{x})$ etc, where $\alpha$ is the growth rate of perturbations with wave number $\lambda$. For a string of cells $\lambda=2 \pi / \omega$; for squares and hexagons $\lambda=\left(\lambda_{1}, \lambda_{2}\right)$, where $\lambda_{1}=2 \pi / \omega_{1}$ and $\lambda_{2}=2 \pi / \omega_{2}$ are the wave lengths for the horizontal and vertical directions, respectively. Substitution into the linearized equations and collecting terms in matrix form gives

$$
M(\alpha ; \lambda)\left(\bar{a}_{1}, . ., \bar{a}_{N}, \bar{f}_{1}, \ldots, \bar{f}_{N}, \bar{b}_{1}, \ldots, \bar{b}_{N}\right)^{T}=0 .
$$

The eigenvalues $\alpha(\lambda)$ of $M(\alpha ; \lambda)$ determine the stability of the homogeneous steady state. The steady state $\left(a_{e}, f_{e}, b_{e}\right)$ must be stable to spatially uniform perturbations, that is all $\alpha(\lambda)$ in (9) have $\operatorname{Re} \alpha(0)<0$. For patterning, this state must become unstable to spatial disturbances, that is $\operatorname{Re} \alpha(\lambda)>0$ for some $\lambda \neq 0$. 


\section{A.1 String of cells}

For a one dimensional string, in which each cell has left and right sides represented by subscripts $l$ and $r$ respectively, we have $\Delta\left(u_{l}\right)=u_{r}-u_{l}=-\Delta\left(u_{r}\right)$ and

$$
M(\alpha ; \lambda)=\left(\begin{array}{cccc}
-k_{a} f_{e}-d_{a}-D_{a}-\alpha & D_{a} & 0 & \cdots \\
D_{a} & -k_{a} f_{e}-d_{a}-D_{a}-\alpha & -k_{a} a_{e} e^{i \lambda} & \cdots \\
0 & -k_{a} f_{e} e^{-i \lambda} & -k_{a} a_{e}-d_{f}-D_{f}-\alpha & \cdots \\
-k_{a} f_{e} e^{i \lambda} & 0 & D_{f}-\alpha & \cdots \\
0 & k_{a} f_{e} e^{-i \lambda} & k_{a} a_{e} & \cdots \\
k_{a} f_{e} e^{i \lambda} & 0 & 0 & \cdots \\
-k_{a} a_{e} e^{-i \lambda} & \frac{\mathcal{A}}{2}+\frac{\gamma_{a} P_{a}\left(2 b_{e}\right)}{4 b_{e}} & \frac{\mathcal{A}}{2}-\frac{\gamma_{a} P_{a}\left(2 b_{e}\right)}{4 b_{e}+k_{d} e^{-i \lambda}} \\
0 & k_{d} e^{i \lambda}+\frac{\mathcal{A}}{2}-\frac{\gamma_{a} P_{a}\left(2 b_{e}\right)}{4 b_{e}} & \frac{\mathcal{A}}{2}+\frac{\gamma_{a} P_{a}\left(2 b_{e}\right)}{4 b_{e}} \\
D_{f} & k_{d}+\frac{\mathcal{F}}{2}+\frac{\gamma_{f} P_{f}\left(2 b_{e}\right)}{4 b_{e}} & \frac{\mathcal{F}}{2}-\frac{\gamma_{f} P_{f}\left(2 b_{e}\right)}{4 b_{e}} \\
-k_{a} a_{e}-d_{f}-D_{f}-\alpha & \frac{\mathcal{F}}{2}-\frac{\gamma_{f} P_{f}\left(2 b_{e}\right)}{4 b_{e}} & \frac{\mathcal{F}}{2}+k_{d}+\frac{\gamma_{f} P_{f}\left(2 b_{e}\right)}{4 b_{e}} \\
0 & -k_{d}-k_{i}-\alpha & 0 \\
k_{a} a_{e} & 0 & -k_{d}-k_{i}-\alpha
\end{array}\right) .
$$

Expanding the determinant gives a sixth order characteristic equation, $P(\alpha, \lambda)$. All the coefficients of $P(\alpha, \lambda)$ are real, and the wave number $\lambda$ is mapped to $K=\cos (\lambda) \in[-1,1]$ - note that the same mapping is observed for strings in the original model.

\section{Stability to homogeneous perturbations}

We first consider where the homogeneous equilibrium is stable in the absence of any spatial effects. This is equivalent to imposing $\lambda=0$, which gives $K=1 . P(\alpha, K=1)$ can be written as a product of two cubic polynomials in $\alpha$, which we denote $q(\alpha)$ and $r(\alpha ; \mathcal{A}, \mathcal{F})$, given by

$$
r(\alpha ; \mathcal{A}, \mathcal{F})=\alpha^{3}+a_{1} \alpha^{2}+a_{2}(\mathcal{A}, \mathcal{F}) \alpha^{1}+a_{3}(\mathcal{A}, \mathcal{F})
$$

where

$$
\begin{gathered}
a_{1}=k_{a} f_{e}+k_{a} a_{e}+d_{a}+d_{f}+k_{i}+k_{d}>0 \\
a_{2}(\mathcal{A}, \mathcal{F})=k_{a} f_{e}\left(d_{f}+k_{i}-\mathcal{A}\right)+k_{a} a_{e}\left(d_{a}+k_{i}-\mathcal{F}\right)+\left(d_{a}+d_{f}\right)\left(k_{d}+k_{i}\right)+d_{a} d_{f} \\
a_{3}(\mathcal{A}, \mathcal{F})=d_{a} k_{a} a_{e}\left(k_{i}-\mathcal{F}\right)+d_{f} k_{a} f_{e}\left(k_{i}-\mathcal{A}\right)+d_{f} d_{f}\left(k_{i}+k_{d}\right),
\end{gathered}
$$


and $q(\alpha)=\alpha^{3}+b_{1} \alpha^{2}+b_{2} \alpha^{1}+b_{3}$, where

$$
\begin{aligned}
b_{1}= & a_{1}+2\left(D_{f}+D_{a}\right) \\
b_{2}= & a_{2}(\mathcal{A}=0, \mathcal{F}=0)+4 D_{a} D_{f} \\
& +2 D_{f}\left(k_{a} f_{e}+d_{a}+k_{d}+k_{i}\right)+2 D_{a}\left(k_{a} a_{e}+d_{f}+k_{d}+k_{i}\right) \\
b_{3}= & a_{3}(\mathcal{A}=0, \mathcal{F}=0)+4 D_{a} D_{f}\left(k_{i}+k_{d}\right) \\
& +2 D_{f}\left(k_{a} f_{e} k_{i}+d_{a}\left(k_{d}+k_{i}\right)\right)+2 D_{a}\left(k_{a} a_{e} k_{i}+d_{f}\left(k_{d}+k_{i}\right)\right) .
\end{aligned}
$$

All the coefficients of $q(\alpha)$ are positive, so that all roots of this cubic have negative real part. It remains to investigate conditions on $r(\alpha ; \mathcal{A}, \mathcal{F})$ for stability of the homogeneous steady state. All the roots of $r(\alpha ; \mathcal{A}, \mathcal{F})$ have negative real part if $a_{1}>0, a_{3}(\mathcal{A}, \mathcal{F})>0$ and $a_{1} a_{2}(\mathcal{A}, \mathcal{F})-a_{3}(\mathcal{A}, \mathcal{F})>0$. The first of these always holds, and the remaining two conditions define lines in the $(\mathcal{A}, \mathcal{F})$ parameter space which delimit the region of stability. Setting $a_{3}(\mathcal{A}, \mathcal{F})=0$ and $a_{1} a_{2}(\mathcal{A}, \mathcal{F})-a_{3}(\mathcal{A}, \mathcal{F})=0$, gives respectively

$$
\begin{aligned}
T_{1}: \quad F= & k_{i}+\frac{d_{f}\left(k_{i}+k_{d}\right)}{k_{a} a_{e}}+\frac{k_{i} f_{e} d_{f}}{d_{a} a_{e}}-\frac{d_{f} f_{e} \mathcal{A}}{d_{a} a_{e}} \\
H_{1}: \quad F= & k_{i}+d_{a}+\frac{d_{f} f_{e}}{a_{e}}+\frac{d_{a} d_{f}+\left(d_{a}+d_{f}\right)\left(k_{d}+k_{i}\right)}{k_{a} a_{e}} \\
& +\frac{d_{a}^{2}\left(d_{f}+k_{d}+k_{i}\right)+d_{a} k_{a}\left(d_{f} f_{e}+d_{a} a_{e}\right)+k_{a} k_{i} f_{e}\left(a_{1}-d_{f}\right)}{k_{a} a_{e}\left(a_{1}-d_{a}\right)} \\
& -\frac{f_{e}\left(a_{1}-d_{f}\right) \mathcal{A}}{a_{e}\left(a_{1}-d_{a}\right)} .
\end{aligned}
$$

$T_{1}$ and $H_{1}$ have negative slope and intersect the axis at a positive value of $\mathcal{F}$. The steady state $\left(a_{e}, f_{e}, b_{e}\right)$ is stable if $\mathcal{F}$ lies below both of these lines. These lines are exactly the same as those found for homogeneous stability in the original L-R model (Owen and Sherratt, 1998; Webb and Owen, 2003).

\section{Instability to inhomogeneous perturbations}

We now consider where the steady state is unstable for some $\lambda \neq 0$. Recall that $K \in$ $[-1,1]$ is the cosine of the wavenumber $\lambda$. Thus, for instability to spatial disturbances, we require at least one root of $P(\alpha)$ to have positive real part for some $K$ in this interval. 
First, we consider the case when the bifurcation arises at $K=-1$. This value of $K$ corresponds to imposing $\lambda=\pi$, which gives a period 2 pattern of alternating high and low cell fates. On making this substitution for $K$ in $P(\alpha)$, it can be shown that this equation again decomposes into a product of two cubic equations. It turns out that the same $q(\alpha)$ and $r(\alpha)$ appear, but with $-\mathcal{A}$ substituted in $r(\alpha)$ instead of $\mathcal{A}$. That is, we get

$$
P(\alpha, K=-1)=q(\alpha) r(\alpha ;-\mathcal{A}, \mathcal{F}) .
$$

Since all the roots of $q(\alpha)$ have negative real part, then the conditions for instability are either $a_{3}(-\mathcal{A}, \mathcal{F})<0$ or $a_{1} a_{2}(-\mathcal{A}, \mathcal{F})-a_{3}(-\mathcal{A}, \mathcal{F})<0$. The patterning bifurcations therefore occur on the following lines

$$
\begin{aligned}
T_{-1}: \quad F= & T_{1}(-\mathcal{A})=k_{i}+\frac{d_{f}\left(k_{i}+k_{d}\right)}{k_{a} a_{e}}+\frac{k_{i} f_{e} d_{f}}{d_{a} a_{e}}+\frac{d_{f} f_{e} \mathcal{A}}{d_{a} a_{e}} \\
H_{-1}: \quad F= & H_{1}(-\mathcal{A})=k_{i}+d_{a}+\frac{d_{f} f_{e}}{a_{e}}+\frac{d_{a} d_{f}+\left(d_{a}+d_{f}\right)\left(k_{d}+k_{i}\right)}{k_{a} a_{e}} \\
& +\frac{d_{a}^{2}\left(d_{f}+k_{d}+k_{i}\right)+d_{a} k_{a}\left(d_{f} f_{e}+d_{a} a_{e}\right)+k_{a} k_{i} f_{e}\left(a_{1}-d_{f}\right)}{k_{a} a_{e}\left(a_{1}-d_{a}\right)} \\
& +\frac{f_{e}\left(a_{1}-d_{f}\right) \mathcal{A}}{a_{e}\left(a_{1}-d_{a}\right)},
\end{aligned}
$$

and the equilibrium is unstable to inhomogeneous perturbations when $\mathcal{F}>\min \left\{T_{-1}, H_{-1}\right\}$. Note that the same lines appear in the original model when $K=-1$.

$P(\alpha)$ does not decompose like this for general $K$, so it is difficult to determine all bifurcations analytically for general parameter values. However, there is a special case which is more amenable to analysis, namely for bifurcations when $P(0, \lambda)=0$, which corresponds to a real eigenvalue crossing the imaginary axis. This bifurcation and the lines $H_{ \pm 1}$ appear to give all the biologically relevant conditions for pattern formation in the $\mathcal{A}-\mathcal{F}$ plane.

\section{Regions and Bifurcations for $\mathrm{P}(0)=0$}

$Q(K)=P(0)$ is a quadratic function in $K$. For notational simplicity, we have set the local feedback parameters to zero, $\gamma_{a}, \gamma_{f}=0$. However, similar results follow when these 
are non-zero. $Q(K)=\rho_{1} K^{2}+\rho_{2} K+\rho_{3}$, where

$$
\begin{aligned}
\rho_{1} & =2 k_{a}^{2} f_{e} D_{a} k_{i} a_{e}\left(d_{f} \mathcal{F}+2 D_{f} \mathcal{F}-2 D_{f} k_{i}\right), \\
\rho_{2} & =-k_{a} f_{e} d_{f} \mathcal{A}\left(\left(d_{f}+2 D_{f}\right)\left(d_{a}+2 D_{a}\right)\left(k_{i}+k_{d}\right)\right. \\
& \left.-k_{i} k_{a}\left(a_{e}\left(d_{a}+2 D_{a}\right)+f_{e}\left(d_{f}+2 D_{f}\right)\right)\right), \\
\rho_{3} & =-\left(\left(d_{f}+2 D_{f}\right)\left(d_{a}\left(k_{i}+k_{d}\right)+k_{i} k_{a} f_{e}\right)+k_{a} d_{a} a_{e} k_{i}\right) \\
& \left(\left(d_{a}+2 D_{a}\right)\left(\mathcal{F} k_{a} a_{e}-d_{f}\left(k_{i}+k_{d}\right)-k_{a} k_{i} a_{e}\right)-k_{i} k_{a} d_{f} f_{e}\right) .
\end{aligned}
$$

The condition for homogeneous stability ensures that $Q(1)>0$, which means that the only possible patterning bifurcations from $Q(K)$ are

- $Q(K)$ has a double root from a minimum at some $K_{c} \in[-1,1]: Q\left(K_{c}\right)=Q^{\prime}\left(K_{c}\right)=$ 0 ,

- $Q(K)$ has a maximum, or a minimum for $K_{c} \notin[-1,1]$, and there is a single root at $K=-1$, given by $Q(-1)=0$.

The calculation for the second case has already been done above when analysing $P(-1)=$ 0 - that is, the bifurcation $Q(-1)=0$ occurs on the line $T_{-1}$. We now find conditions on $\mathcal{A}$ and $\mathcal{F}$ for a double root in $[-1,1]$. The critical point of $Q(K)$ is at:

$$
\begin{aligned}
K_{\mathrm{c}}=\frac{d_{f} \mathcal{A}}{4 D_{a} a_{e}\left(\mathcal{F}\left(d_{f}+2 D_{f}\right)-2 D_{f} k_{i}\right)} & \left(\frac{\left(k_{i}+k_{d}\right)\left(2 D_{a}+d_{a}\right)\left(d_{f}+2 D_{f}\right)}{k_{a} k_{i}}\right. \\
& \left.+f_{e}\left(d_{f}+2 D_{f}\right)+a_{e}\left(d_{a}+2 D_{a}\right)\right) .
\end{aligned}
$$

When $\mathcal{F}>T_{\kappa}=2 D_{f} k_{i} /\left(d_{f}+2 D_{f}\right)$, the coefficient of $K^{2}$ is positive, and $K_{\mathrm{c}}$ corresponds to a minimum. This minimum value is in $[-1,1]$ when $\mathcal{A}$ and $\mathcal{F}$ lie in the region bounded by the lines $K_{ \pm 1}$ :

$$
K_{ \pm 1}: \quad \mathcal{F}=\frac{2 D_{f} k_{i}}{d_{f}+2 D_{f}} \mp \frac{\mathcal{A} d_{f}}{4 D_{a}}\left(\frac{\left(d_{a}+2 D_{a}\right)\left(k_{i}+k_{d}\right)}{k_{a} a_{e} k_{i}}+\frac{f_{e}}{a_{e}}+\frac{d_{a}+2 D_{a}}{d_{f}+2 D_{f}}\right) .
$$

Setting the discriminant of the quadratic $Q(K)$ to be equal to zero gives an ellipse in $\mathcal{A}-\mathcal{F}$ space that corresponds to the locus of points where the quadratic has double roots. 
The expression for $T_{d}$ is rather lengthy, and we omit it for brevity. In the case $\gamma_{a}=\gamma_{f}=0$, $T_{d}$ is symmetric about the $\mathcal{F}$-axis. It has a center at:

$$
\mathcal{A}_{\mathrm{c}}=0, \quad \mathcal{F}_{\mathrm{c}}=\frac{k_{i}}{2}+\frac{d_{f}\left(k_{i}+k_{d}\right)}{2 a_{e} k_{a}}+\frac{k_{i} D_{f}}{d_{f}+2 D_{f}}+\frac{k_{i} d_{f} f_{e}}{2 a_{e}\left(d_{a}+2 D_{a}\right)} .
$$

Moreover, straightforward examination shows that $T_{d}$ sits wholly within the upper-half plane, and intersects the $\mathcal{F}$-axis at

$$
\mathcal{F}_{\mathrm{u}}=k_{i}+\frac{d_{f}\left(k_{i}+k_{d}\right)}{a_{e} k_{a}}+\frac{d_{f} f_{e} k_{i}}{a_{e}\left(d_{a}+2 D_{a}\right)}, \quad \text { and } \quad \mathcal{F}_{1}=T_{\kappa}<\mathcal{F}_{\mathrm{u}} .
$$

The ellipse $T_{d}$ intersects the two lines $T_{ \pm 1}$ tangentially - see Figure 4 . In fact, for any fixed $K, Q(K)$ gives a straight line in $\mathcal{A}-\mathcal{F}$ space, and each of these straight line contours are tangent to $T_{d}$. The points at which the two lines $T_{ \pm 1}$ touch the curve $T_{d}$ are, respectively

$$
\begin{aligned}
& \left(\mathcal{A}_{\mathrm{p}}, T_{1}\left(\mathcal{A}_{\mathrm{p}}\right)\right) \text { and }\left(-\mathcal{A}_{\mathrm{p}}, T_{-1}\left(-\mathcal{A}_{\mathrm{p}}\right)\right) \text { where } \\
\mathcal{A}_{p}= & \left\{4 D_{a} k_{i}\left(\left(d_{a}\left(k_{i}+k_{d}\right)+f_{e} k_{a} k_{i}\right)\left(d_{f}+2 D_{f}\right)+k_{a} k_{i} a_{e} d_{a}\right)\right\} \\
& /\left\{2 k_{a} f_{e} k_{i}\left(D_{f}\left(d_{a}+2 D_{a}\right)+D_{a}\left(d_{f}+2 D_{f}\right)\right)\right. \\
& +\left(d_{a} a_{e}+f_{e} d_{f}\right)\left(d_{a}+2 D_{a}\right) k_{i} k_{a} \\
& \left.+d_{a}\left(k_{i}+k_{d}\right)\left(d_{a}+2 D_{a}\right)\left(d_{f}+2 D_{f}\right)\right\} .
\end{aligned}
$$

The minimum point of $Q(K)$ is in $[-1,1]$ when $\mathcal{F}>\max \left\{K_{m}, K_{p}\right\}$, and the corresponding minimum values $Q\left(K_{\mathrm{c}}\right)$ are less than zero when $\mathcal{F}$ lies above the upper part of the ellipse $T_{d}$. See Figure $4(\mathrm{a})$-(c) for a qualitative illustration of these curves in the $(\mathcal{A}, \mathcal{F})$ plane.

So far, we have shown that the pattern region in $\mathcal{A}-\mathcal{F}$ space is bounded by four straight lines $\left(T_{1}, H_{1}\right.$ for stability to homogeneous perturbations and $T_{-1}, H_{-1}$ for instability to inhomogeneous perturbations) and an ellipse $T_{d}$ - which is the same configuration of lines and curves as in the original model. For $d_{f}>d_{a}$, the line $T_{-1}$ is steeper than $H_{-1}$, and they intersect for $\mathcal{F}>0$, with $\mathcal{A}$ taking either sign. For $d_{f}<d_{a}$, the line $H_{-1}$ is steeper than $T_{-1}$, and these two lines intersect for $\mathcal{F}<T_{\kappa}$ and $\mathcal{A}<0$. Thus, there is a region of $\mathcal{A}$ and $\mathcal{F}$ space in which the steady state is unstable to patterns via a Hopf bifurcation, and limit cycle oscillations are predicted; this is when $\mathcal{F}<T_{-1}$ and $\mathcal{F}>H_{-1}$. 


\section{A.2 Square and Hexagonal arrays}

It appears in Figure 5(b) that the pattern region for squares is bounded by the same four straight lines, $T_{ \pm 1}$ and $H_{ \pm 1}$, as strings. In fact, on substituting $\lambda=0$ and $\lambda=\pi$ into $M(\alpha, \lambda)$ for squares, $\operatorname{det}(M)$ simplifies so that the 12 th order polynomial again decomposes, this time into a 9th order part not involving $\mathcal{A}$ and $\mathcal{F}$, and the same cubics $r(\alpha ; \pm \mathcal{A}, \mathcal{F})$ defined by $(10,11)$. Thus, we do indeed get the same bifurcation lines $T_{ \pm 1}, H_{ \pm 1}$.

\section{B The role of intra-membrane protein diffusion and polarity}

\section{B.1 Pattern region}

We can see by looking at (16) that with no intra-membrane ligand diffusion, $D_{a}=0$, $Q(K)$ becomes simply linear, and the ellipse $T_{d}$ is not defined. In this case, the pattern region is smaller, being just the region in $(\mathcal{A}, \mathcal{F})$ space bounded by the four straight lines $T_{ \pm 1}, H_{ \pm 1}$, and in particular there is no pattern formation for activation of ligand production $(\mathcal{A}>0)$. Figure 7 shows how increasing intra-membrane ligand diffusion, $D_{a}$, increases the size of the pattern region and makes pattern formation with lateral induction possible. In addition, intra-membrane receptor diffusion, $D_{f}$, also modulates the patterning region by shifting the ellipse $T_{d}$ along the $\mathcal{F}$-axis.

We now look in detail at how varying these parameters affects the possible the size of the patterning region, namely the possible arrangements of the lines $K_{ \pm 1}$ and the points $\mathcal{F}_{u}, \mathcal{F}_{l}, \mathcal{F}_{c}$ and $\mathcal{A}_{p}$, and the corresponding wavelengths. Recall that the expressions for the lines $T_{ \pm 1}, H_{ \pm 1}$ do not involve $D_{a}$ or $D_{f}$. We consider this case by case below:

- $\mathcal{F}_{u}=\mathcal{F}_{u}\left(D_{a}\right): \mathcal{F}_{u}$ is a decreasing, saturating function of $D_{a} . \mathcal{F}_{u}(0)$ is equal to $T_{ \pm 1}(\mathcal{A}=0)=k_{i}+d_{f}\left(k_{i}+k_{d}\right) / a_{e} k_{a}+d_{f} f_{e} k_{i} / a_{e} d_{a}$ and $\mathcal{F}_{u} \rightarrow k_{i}+d_{f}\left(k_{i}+k_{d}\right) / a_{e} k_{a}$ as $D_{a} \rightarrow \infty$.

- $\mathcal{F}_{l}=\mathcal{F}_{l}\left(D_{f}\right): \mathcal{F}_{l}$ is an increasing, saturating function of $D_{f} ; \mathcal{F}_{l}(0)=0$ and $\mathcal{F}_{l} \rightarrow k_{i}$ as $D_{f} \rightarrow \infty$. 
- $\mathcal{F}_{c}=\mathcal{F}_{c}\left(D_{a}, D_{f}\right)$ : is an increasing (decreasing), saturating function of $D_{f}\left(D_{a}\right)$; $\mathcal{F}_{c} \rightarrow k_{i}+d_{f}\left(k_{i}+k_{d}\right) / 2 a_{e} k_{a}$ as $D_{a}, D_{f} \rightarrow \infty$.

- $K_{ \pm 1}=K_{ \pm 1}\left(D_{a}, D_{f}\right)$ : both of these lines intersect the $\mathcal{F}$-axis at $\mathcal{F}_{l}$. When $D_{a}=0$, they both coincide with the vertical line $\mathcal{A}=0$, and their slopes become more shallow as $D_{a}$ and $D_{f}$ increase.

- $\mathcal{A}_{p}=\mathcal{A}_{p}\left(D_{a}, D_{f}\right): \mathcal{A}_{p}$ is an increasing (decreasing), saturating function of $D_{a}\left(D_{f}\right)$; $\mathcal{A}_{p}\left(D_{a}=0\right)=0$ and $\mathcal{A}_{p} \rightarrow\left(2 k_{i}^{2}\left(k_{a} f_{e}+d_{a}\right)+2 d_{a} k_{d}\right) /\left(k_{i}\left(2 k_{a} f_{e}+d_{a}\right)+d_{a} k_{d}\right)$ as $D_{a}, D_{f} \rightarrow \infty$.

These results are summarised in Table 1, and illustrated in Figure 7 - part (a) shows the pattern region when $D_{a}=0$; parts (b) and (c) show the effect of increasing $D_{a}$, with $D_{f}$ fixed; and, parts (d)-(f) show the effect of increasing $D_{f}$. In each case, the pattern region is shaded.

We now consider how altering the bias in ligand and receptor production, $\gamma_{a}, \gamma_{f} \neq 0$, affects patterning. For biased receptor production, the centre and bottom of the ellipse are shifted along the $\mathcal{F}$-axis. In particular, for a string of cells, the expressions for its centre and intersections with the $\mathcal{F}$-axis are now, respectively,

$$
\mathcal{A}_{\mathrm{c}}=0, \quad \mathcal{F}_{\mathrm{c}}=\mathcal{F}_{\mathrm{c}}+\frac{d_{f} \gamma_{f} P_{f}\left(2 b_{e}\right)}{4 b_{e}\left(d_{f}+2 D_{f}\right)}
$$

and

$$
\mathcal{F}_{\mathrm{u}}=\mathcal{F}_{\mathrm{u}}, \quad \text { and } \quad \mathcal{F}_{\mathrm{l}}=T_{\kappa}+\frac{d_{f} \gamma_{f} P_{f}\left(2 b_{e}\right)}{2 b_{e}\left(d_{f}+2 D_{f}\right)} .
$$

However, when biased ligand production is included, $\gamma_{a} \neq 0$, the ellipse loses its symmetry about the $\mathcal{F}$-axis and these expressions do not simplify so easily. Instead, we see by looking at Figure 9 that biased ligand production modulates the patterning region by tilting the ellipse. The pattern region for $\mathcal{A}>0$ becomes smaller when $\gamma_{a}>0$, and larger for $\gamma_{a}<0$ (compare the dashed and solid ellipses in Figure 9 - dashed ellipses show how the patterning bifurcations move when $\gamma_{a}= \pm 1$ ).

\section{Regular polarity requires biased production}

Consider opposite sides of a single $N$ sided cell, labelled by subscripts $l$ and $r$ for left and right, with periodic boundary conditions, and without biased production or intra- 
membrane diffusion. The steady state equations yield

$$
f_{j}=\frac{\left(k_{d}+k_{i}\right) b_{j}}{k_{a} a_{i}}, \quad i, j \in\{r, l\} .
$$

Substituting these expressions into the equations for ligand and free receptor on these sides, gives four equations for $a_{r}, a_{l}$ and $b_{r}, b_{l}$. When the single cell system is at equilibrium, these are given by

$$
\begin{aligned}
& 0=-k_{i} b_{j}-d_{a} a_{i}+\frac{P_{a}\left(b_{r}+b_{l}\right)}{2} \\
& 0=-k_{i} b_{i}-d_{f} \frac{\left(k_{d}+k_{i}\right) b_{i}}{k_{a} a_{j}}+\frac{P_{f}\left(b_{r}+b_{l}\right)}{2} .
\end{aligned}
$$

Rearranging the equation (26) for $i, j=r, l$, and vice-versa, we obtain the expression:

$$
k_{i}\left(b_{r}-b_{l}\right)=d_{a}\left(a_{r}-a_{l}\right)
$$

We are interested in solutions with $u_{r} \neq u_{l}$, for $u=a, f$ or $b$, and without loss of generality we can assume that $b_{r}>b_{l}$. We therefore require that $a_{r}>a_{l}$. To find another relation, we now rearrange equation (27) for $i, j \in\{r, l\}$, to give:

$$
k_{i} b_{l}+d_{f} \frac{\left(k_{d}+k_{i}\right) b_{l}}{k_{a} a_{r}}=k_{i} b_{r}+d_{f} \frac{\left(k_{d}+k_{i}\right) b_{r}}{k_{a} a_{l}}
$$

Since $b_{r}>b_{l}$ and $a_{r}>a_{l}$, then $b_{r} / a_{l}>b_{l} / a_{r}$, so that the right-hand side of this expression is always larger than the left-hand side, and therefore stationary solutions with $u_{r} \neq u_{l}$ do not exist. The same result follows in a string of cells even when intra-membrane diffusion is included, but the effect of diffusion on cells with more than 2 sides is unclear. It is possible that for $N$ large, rapid diffusion in one protein, ligand say, and slow diffusion in receptor, can lead to a Turing instability to a polarized pattern on a single cell, and this behaviour has indeed been observed in a continuous activator-inhibitor model for Min proteins in E.Coli (Meinhardt and de Boer, 2001). However, without such diffusion or biased production, regular polarity on a length scale of a single cell is not possible with this ligand-receptor mechanism.

\section{Parameter values}

In our numerical simulations, we use parameter values based on experimental data for binding of epidermal growth factor to its receptors. Unless otherwise stated, the rate constants are taken as: $k_{a}=1.8 \times 10^{8} \mathrm{M}^{-1} \mathrm{~min}^{-1}, k_{d}=0.12 \mathrm{~min}^{-1}, k_{i}=0.019 \mathrm{~min}^{-1}$, 
$d_{a}=0.03 \mathrm{~min}^{-1}$, and $d_{f}=0.01 \mathrm{~min}^{-1}$. An explanation of the choice of each individual value can be found in the work of Owen \& Sherratt (Owen and Sherratt, 1998).

A typical cell diameter is around $10 \mu \mathrm{m}$, giving a volume of $10^{3}(\mu \mathrm{m})^{3}=10^{-12}$ litre for a cubic cell, and thus $10^{12}$ cells per litre. Molarity, M, can be written as moles/litre, or $6 \times 10^{23}$ molecules/litre, or alternatively $\mathrm{M}=6 \times 10^{23}$ (molecules/cell)(cell/litre) $=6 \times$ $10^{11}$ molecules/cell, with which $k_{a}$ becomes 0.0003 molecules ${ }^{-1}$ cell $\mathrm{min}^{-1}$. For the sides model, we write $k_{a}=0.0003$ (molecules ${ }^{-1}$ side $\min ^{-1}$ ) (cell/side), the last term being simply $1 / N$, where $N$ is the number of sides per cell, and thus $k_{a}=0.0003 \times N$ molecules $^{-1}$ side $\min ^{-1}$.

The production terms include a combination of local and global responses to binding:

$$
P_{u}(b,\langle b\rangle)=\frac{\check{P}_{u}(\langle b\rangle)}{N}\left(1-\gamma_{u} \frac{\langle b\rangle-N b}{\langle b\rangle}\right),
$$

where $\check{P}_{u}(\langle b\rangle)$ is the total production for the cell, $\gamma_{u}$ is a homotopy parameter used to vary the relative contribution of the local and global responses. We require the production terms in (30) to be non-negative, that is

$$
1-\gamma_{u} \frac{\langle b\rangle-N b}{\langle b\rangle} \geq 0
$$

where $b$ is the number of bound receptors on a particular membrane segment, $\langle b\rangle$ is the total number of bound receptors on the whole cell and $N$ is the number of sides. If $b \lessgtr\langle b\rangle / N$, then this inequality requires that $\gamma_{u}$ satisfies

$$
\gamma_{u} \lesseqgtr \frac{\langle b\rangle}{\langle b\rangle-N b} .
$$

The limiting cases for these are when $b=0$ and $b=\langle b\rangle$, respectively, which upon substitution into (32) implies that

$$
\gamma_{u} \in\left[\frac{-1}{N-1}, 1\right]
$$

putting $\gamma_{u}$ in $[-1,1],[-1 / 3,1]$, and $[-1 / 5,1]$ for strings, squares and hexagons. Recall that $\gamma_{u}=0$ gives unbiased global production; $\gamma_{u}>0$ enhances production on the side with the most bound receptors; and $\gamma_{u}<0$ gives an opposite bias.

The production functions $\check{P}_{u}(\langle b\rangle)$ are of Hill form such that

$$
\check{P}_{a}(\langle b\rangle)=\frac{C_{1}\langle b\rangle^{m}}{C_{2}^{m}+\langle b\rangle^{m}}, \quad \text { and } \quad \check{P}_{f}(\langle b\rangle)=C_{3}+\frac{C_{4}\langle b\rangle^{n}}{C_{5}^{n}+\langle b\rangle^{n}} .
$$


Note that inhibition is given by choosing negative exponents - this is equivalent to the more usual decreasing Hill function form $\check{P}_{a}(\langle b\rangle)=C_{1} C_{2}^{h} /\left(C_{2}^{h}+\langle b\rangle^{h}\right)$ where $h=-m$, and similarly for $\check{P}_{f}(\langle b\rangle)$.

Some of the parameters $C_{1}-C_{5}$ can be specified since the functions $\check{P}_{a}$ and $\check{P}_{f}$ must satisfy at least two experimentally testable conditions. For example, in the absence of ligand there will be some background level of receptor expression. This is one of the homogeneous steady states of the model (1), giving

$$
\check{P}_{f}(0)=C_{3}=N d_{f} r_{0}
$$

where $r_{0}$ is the unstimulated receptor number. Normal equilibrium levels of free and bound receptors, say $f_{e}$ and $b_{e}$, are often known in particular systems. With these values, the steady state equation of (1c) yields the normal steady state level of free ligand, $a_{e}$, and $(1 \mathrm{a}, \mathrm{b})$ give the values of the feedback functions at this steady state, that is

$$
a_{e}=\frac{\left(k_{d}+k_{i}\right) b_{e}}{k_{a} f_{e}}, \quad \check{P}_{a}\left(N b_{e}\right)=N\left(k_{i} b_{e}+d_{a} a_{e}\right) \quad \text { and } \quad \check{P}_{f}\left(N b_{e}\right)=N\left(k_{i} b_{e}+d_{f} f_{e}\right) .
$$

The latter two conditions fix the parameters $C_{1}$ and $C_{4}$, to give

$$
C_{1}=N\left(k_{i} b_{e}+d_{a} a_{e}\right)\left(\beta_{a}^{m}+1\right), \quad \text { and } \quad C_{4}=N\left(k_{i} b_{e}+d_{f}\left(f_{e}-r_{0}\right)\right)\left(\beta_{f}^{m}+1\right),
$$

where we have used $C_{2}=N \beta_{a} b_{e}$ and $C_{5}=N \beta_{f} b_{e}$. Substituting these expressions into (34) and differentiating, gives

$$
\mathcal{A} \equiv P_{a}^{\prime}\left(N b_{e}\right)=\frac{m\left(k_{i} b_{e}+d_{a} a_{e}\right) \beta_{a}^{m}}{b_{e}\left(\beta_{a}^{m}+1\right)}, \quad \mathcal{F} \equiv P_{f}^{\prime}\left(N b_{e}\right)=\frac{n\left(k_{i} b_{e}+d_{f}\left(f_{e}-r_{0}\right)\right) \beta_{f}^{n}}{b_{e}\left(\beta_{f}^{n}+1\right)} .
$$

$\mathcal{A}$ and $\mathcal{F}$ are the key parameters in the control of pattern formation. By fixing $\beta_{a}$ and $\beta_{f}, m$ can be used to vary $\mathcal{A}$ and $n$ to vary $\mathcal{F}$. If $\beta_{a}=\beta_{f}=1, \mathcal{A}$ and $\mathcal{F}$ are linear functions of $m$ and $n$. $\beta_{a}, \beta_{f}<1, \mathcal{A}$ and $\mathcal{F}$ are strictly increasing for $m<0$, they each have a maximum for some $m>0$, and then tend to zero as $m$ gets large. For $\beta_{a}, \beta_{f}>1$, $\mathcal{A}$ and $\mathcal{F}$ are strictly increasing for $m>0$, and have a turning point for some $m<0$. Unless otherwise stated, we take $\beta_{a}=\beta_{f}=1$. We assume that the unstimulated receptor number, $r_{0}$, and equilibrium levels of free and bound receptors, $f_{e}$ and $b_{e}$, are all 3000 per cell, or equivalently $3000 / N$ per side.

We vary the intra-membrane diffusion coefficients $D_{a}$ and $D_{f}$ in our analysis and calculations. If a cell has $N$ sides of length $l$ then the total length of membrane is $L=N l$, and rescaling space by $L$ we have

$$
D_{u} u_{x x} \approx \frac{D_{u}}{L^{2}} \frac{u_{i+1}-2 u_{i}+u_{i-1}}{l^{*^{2}}}=D_{u}^{*}\left(u_{i+1}-2 u_{i}+u_{i-1}\right),
$$


where we use the fact that $l^{*}=1 / N$ and define

$$
D_{u}^{*}=\frac{N^{2} D_{u}}{L^{2}}=\frac{D_{u}}{l^{2}}
$$

Thus, transport between sides is given by

$$
D_{u}^{*} \Delta^{*}\left(u_{i}\right)=D_{u}^{*}\left(u_{i+1}-2 u_{i}+u_{i-1}\right)
$$

and dropping the *'s gives the form in the main text.

Lauffenberger and Linderman give a range of diffusion coefficients for transmembrane receptors of $10^{-11}-10^{-9} \mathrm{~cm}^{2} s^{-1}=0.06-6 \mu \mathrm{m}^{2} \mathrm{~min}^{-1}$ (Lauffenburger and Linderman, 1996). If we assume that transmembrane ligand has a similar range of diffusivity, and consider sides of length $5 \mu \mathrm{m}$, this gives ranges for the dimensionless parameters $D_{u}^{*}$ of $0.0024-$ 0.24 .

\section{E Irregular arrays}

For irregular arrays it is no longer convenient to consider numbers of molecules per side, but rather molecules per unit length on a given side. The form of the model equations is precisely (1), but the functional forms and transport terms are slightly modified as follows. First, we define $\langle b\rangle$ to be the average bound receptor density, given by the total number of bound receptors on a cell divided by the total length of membrane:

$$
\langle b\rangle=\frac{\sum_{i=1}^{N} l_{i} b_{i}}{\sum_{i=1}^{N} l_{i}} .
$$

Since we are now dealing with concentrations, we need not distribute production terms by dividing by the number of sides, and the redistribution term alters in the light of the above definition of average density:

$$
P_{u}(b,\langle b\rangle)=\check{P}_{u}(\langle b\rangle)\left(1-\gamma_{u} \frac{\langle b\rangle-b}{\langle b\rangle}\right) .
$$

Thus, if a side of a cell is at the average bound receptor density for that cell, it receives no bias in production.

Finally, the transport terms are altered to take account of the lengths of the sides:

$$
D_{u} \Delta\left(u_{i}\right)=D_{i}^{+}\left(u_{i+1}-u_{i}\right)+D_{i}^{-}\left(u_{i-1}-u_{i}\right)
$$


with

$$
D_{i}^{+}=\frac{8 D_{u}}{\left(l_{i}+l_{i+1}\right)\left(l_{i-1}+2 l_{i}+l_{i+1}\right)} \quad \text { and } \quad D_{i}^{-}=\frac{8 D_{u}}{\left(l_{i-1}+l_{i}\right)\left(l_{i-1}+2 l_{i}+l_{i+1}\right)} .
$$

When $l_{i} \equiv l=L / N, D_{i}^{ \pm}=D_{u} / l^{2}$ and $\langle b\rangle=\left(\sum_{i=1}^{N} b_{i}\right) / N$, so rescaling $a, f$ and $b$ by $N$ leads to the system for regular sides. 


\section{Tables}

Table 1: Bifurcation lines and curve for strings of cells in the limit $D_{a}, D_{f} \rightarrow \infty$ (right column), and the corresponding expressions derived in (Webb and Owen, 2003) for homogeneous cells (left column). We remark that a formal reduction may be made if intramembrane bound receptor diffusion, $D_{b}$, is also included and the limit $D_{a}, D_{f}, D_{b} \rightarrow \infty$ is considered. However, this seems highly unlikely given that bound receptors are connected to two cells via ligand.

\begin{tabular}{|l|l|l|}
\hline & $\begin{array}{l}\text { Homogeneous cell (Webb } \\
\text { and Owen, 2003) }\end{array}$ & Sides model $\left(D_{a}, D_{f} \rightarrow \infty\right)$ \\
\hline$F_{u}$ & $\mathcal{F}=k_{i}+\frac{d_{f}\left(k_{i}+k_{d}\right)}{a_{e} k_{a}}$ & $\mathcal{F}=k_{i}+\frac{d_{f}\left(k_{i}+k_{d}\right)}{a_{e} k_{a}}$ \\
\hline$F_{c}$ & $\mathcal{F}=k_{i}+\frac{d_{f}\left(2 k_{d}+k_{i}\right)}{2 a_{e} k_{a}}$ & $\mathcal{F}=k_{i}+\frac{d_{f}\left(k_{i}+k_{d}\right)}{2 a_{e} k_{a}}$ \\
\hline$F_{l}$ & $\mathcal{F}=k_{i}+\frac{d_{f} k_{d}}{a_{e} k_{a}}$ & $\mathcal{F}=k_{i}$ \\
\hline$A_{p}$ & $\mathcal{A}=\frac{2 k_{i}\left(k_{a} f_{e}+d_{a}\right)}{2 k_{a} f_{e}+d_{a}}$ & $\mathcal{A}=\frac{2 k_{i}^{2}\left(k_{a} f_{e}+d_{a}\right)+2 d_{a} k_{d}}{k_{i}\left(2 k_{a} f_{e}+d_{a}\right)+d_{a} k_{d}}$ \\
\hline$K_{1,-1}$ & $\mathcal{F}=k_{i}+\frac{d_{f} k_{d}}{a_{e} k_{a}} \pm \frac{d_{f} \mathcal{A}}{2 k_{a} a_{e}}$ & $\mathcal{F}=k_{i} \pm \frac{d_{f} \mathcal{A}\left(k_{i}+k_{d}\right)}{2 k_{i} k_{a} a_{e}}$ \\
\hline$T_{d}$ & $\mathcal{F}=k_{i}+\frac{d_{f}\left(2 k_{d}+k_{i}\right)}{2 k_{a} a_{e}}$ & $\mathcal{F}=k_{i}+\frac{d_{f}\left(k_{d}+k_{i}\right)}{2 k_{a} a_{e}}$ \\
$\pm \frac{d_{f}}{2 k_{a} a_{e}} \sqrt{k_{i}^{2}-\frac{k_{a} f_{e} A^{2}}{d_{a}+k_{a} f_{e}}}$ & $\begin{array}{l} \pm \\
\frac{d_{f}\left(k_{i}+k_{d}\right)}{2 k_{a} a_{e} k_{i}} \sqrt{k_{i}^{2}-\frac{k_{a} f_{e} k_{i} A^{2}}{\left(d_{a}+k_{a} f_{e}\right) k_{i}+d_{a} k_{d}}}\end{array}$ \\
\hline
\end{tabular}




\section{Figures \& Legends}

(a) Squares

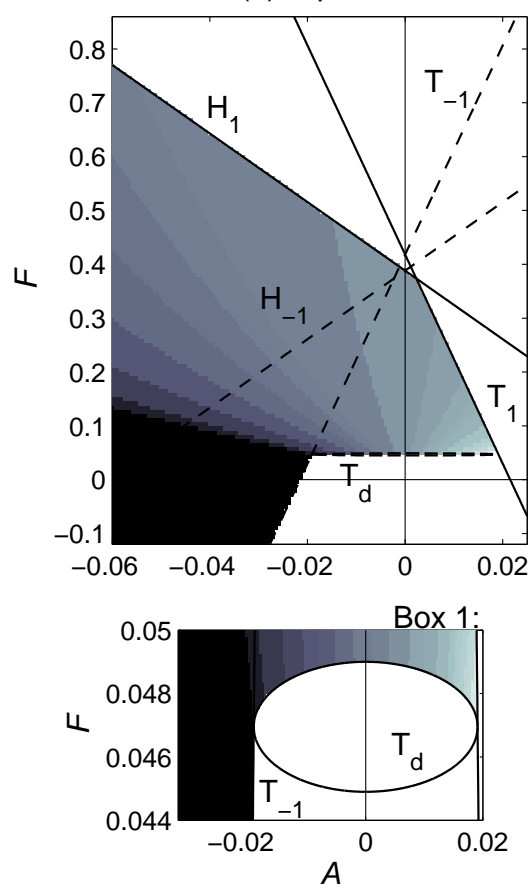

(b) Hexagons

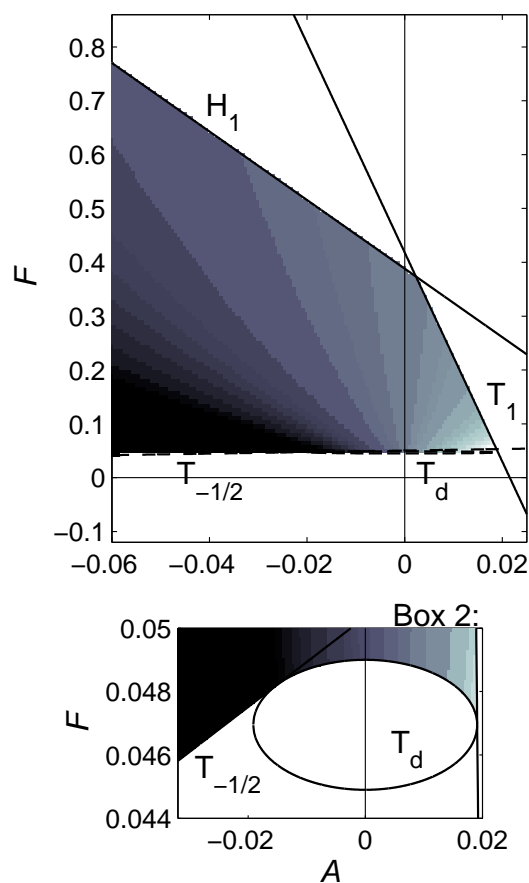

Figure 1: Patterning bifurcations and fastest growing modes for the L-R model of (Webb and Owen, 2003), in which protein distributions are homogeneous on each cell. Part (a) and box 1 are for arrays of square cells; part (b) and box 2 are for hexagons the picture for strings is similar to that for squares. This classification is determined by linear stability analysis of the homogeneous equilibrium, with parameters $\mathcal{A}$ and $\mathcal{F}$ representing the slopes of the ligand and receptor production terms $\left(P_{a}^{\prime}\right.$ and $\left.P_{f}^{\prime}\right)$ at this steady state. The pattern region is bounded by four straight lines $T_{1}, H_{1}, T_{\kappa}, H_{\kappa}$ and an ellipse, $T_{d}$, that intersects the lines $T_{1}$ and $T_{\kappa}$ tangentially (see zoom boxes) $-\kappa=-1$ for strings/squares and $\kappa=-1 / 2$ for hexagons. Wavelengths increase from left to right (black is for wavelength 2 in strings/squares and 3 in hexagons; light grey indicates longer wavelengths). Lateral inhibition on ligand can support wavelengths longer than two provided receptor up-regulation is included. Parameter values and expressions for $T_{1}, H_{1}, T_{\kappa}, H_{\kappa}$ and $T_{d}$ are given in (Webb and Owen, 2003). 
(a)

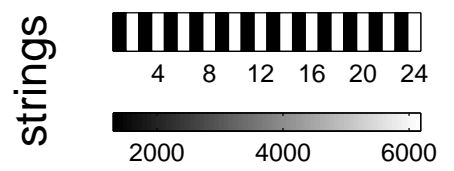

(d)

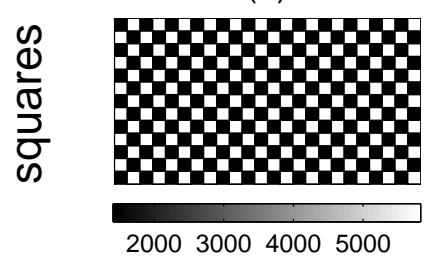

(g)

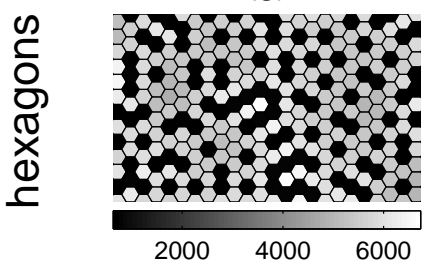

(b)

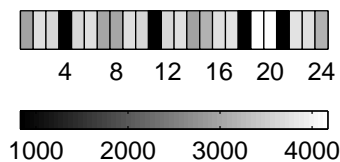

(e)

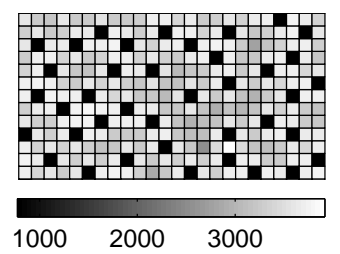

(h)

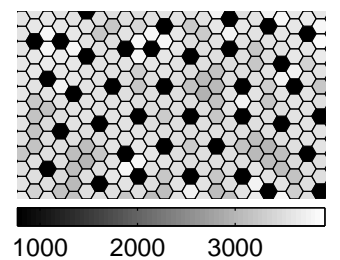

(c)

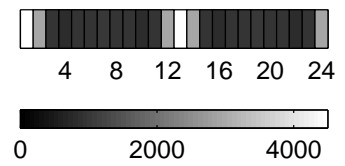

(f)

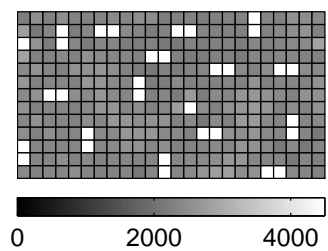

(i)

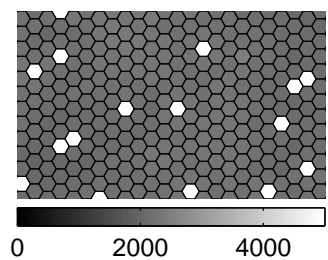

Figure 2: Numerical simulations of the juxtacrine model (Webb and Owen, 2003), solved on a string of 24 cells (a-c), and $24 \times 24$ arrays of square cells (d-f) and hexagonal cells (g-i). For clarity, in (d-i) we show a subset of the full array. We plot the densities of bound receptors arising from small random perturbations of the homogeneous steady state. The boundary conditions are periodic. Strong lateral inhibition (first column) tends to give short range patterns, weaker inhibition (second column) gives longer wavelengths, while lateral induction (third column) can give patterns with much longer wavelengths. The parameters are the same as in (Webb and Owen, 2003), except that in (c) $m=1.1, n=$ $2.5, C_{2}=3900$, (f) $m=1.8, n=3, C_{2}=2500$, (i) $m=1.8, n=3, C_{2}=2500$. 
b)
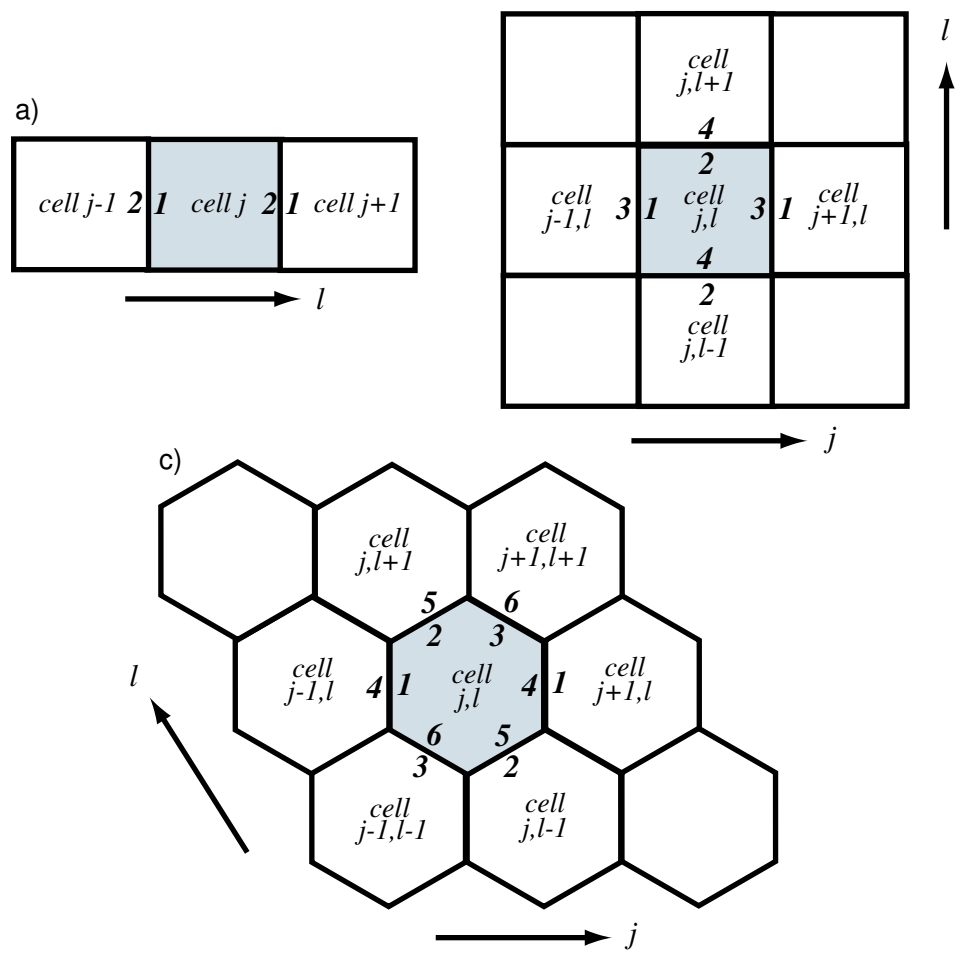

Figure 3: We consider protein and receptor distributions on each side of a cell in onedimensional linear arrays and two dimensional arrays of squares and hexagons. Interactions between cells occur on adjacent sides of neighbouring cells. Here we indicate the spatial labelling schemes, with bold numerals corresponding to the index of each side. 
(a)

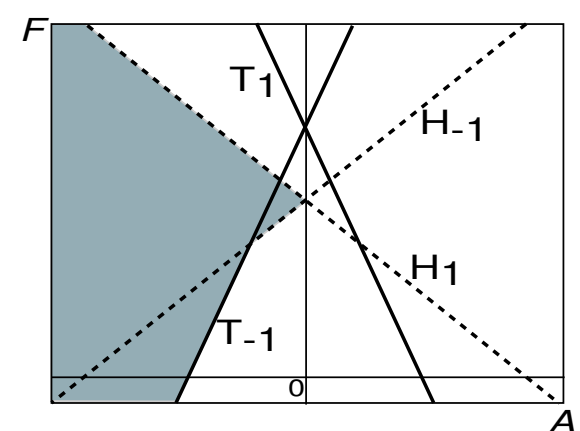

(b)

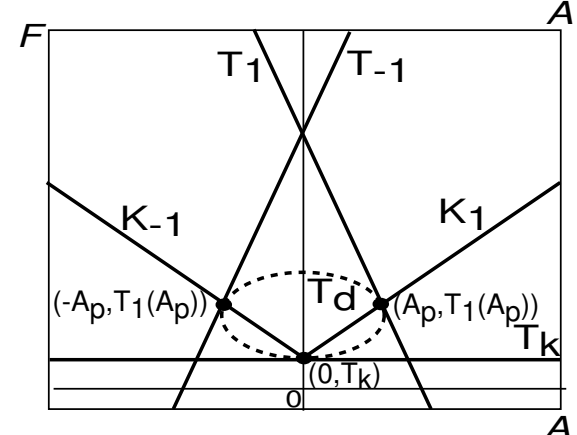

(c)

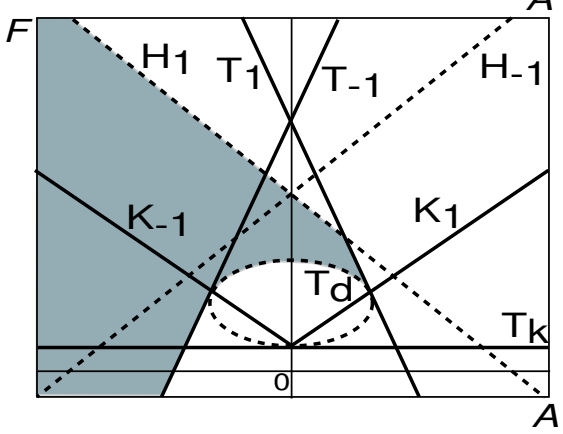

Figure 4: Lines and curves in $(\mathcal{A}, \mathcal{F})$ space that delimit the region for pattern formation in strings of cells for the model (1). Below the lines $T_{1}$ and $H_{1}$, the homogeneous equilibrium is stable to homogeneous perturbations. (a) With no intra-membrane ligand diffusion, $D_{a}=0$, the patterning bifurcation can only occur with wavelength two, along the lines $T_{-1}$ and $H_{-1}$. (b) With $D_{a} \neq 0$, patterning can also arise via the ellipse $T_{d}$, which touches the lines $T_{ \pm 1}$ tangentially at $\left( \pm A_{p}, T_{1}\left(A_{p}\right)\right)$, respectively. Pattern formation is possible in the shaded areas in (c), and for unshaded regions there is no patterning instability. Note that when $D_{a}=0$ (part (a)), it is possible to cross directly to patterns through a Hopf bifurcation for $\mathcal{F}>0$ via the line $H_{-1}$. Expressions for the indicated lines and curves are derived in Appendix A. 
(a) String

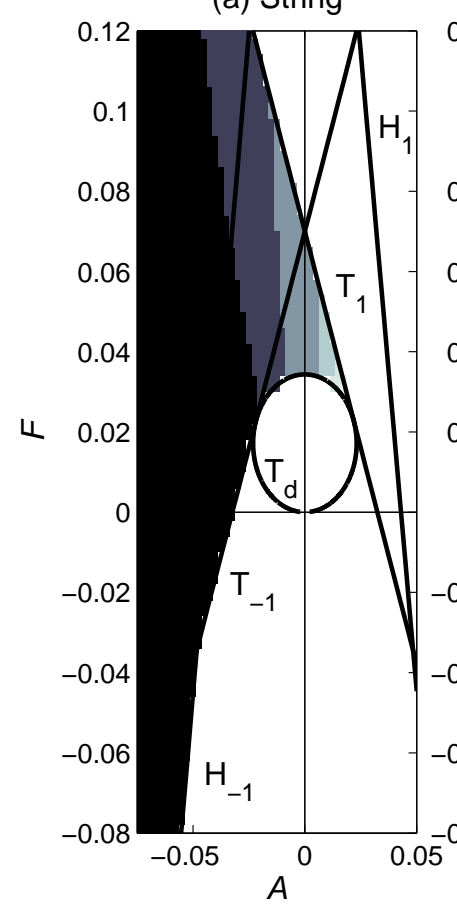

(b) Squares

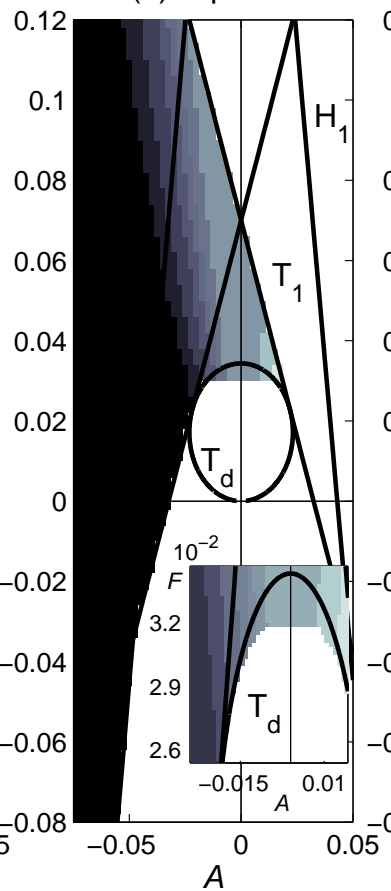

(c) Hexagons

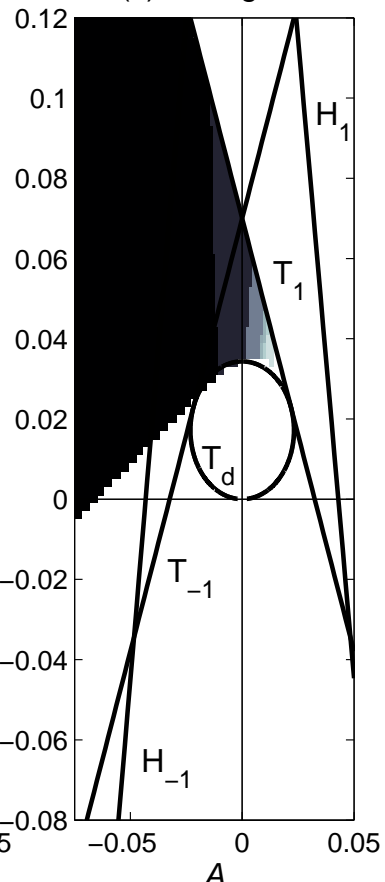

Figure 5: Examples of patterning bifurcations and fastest growing modes found via numerical calculations of stability for steady states of (1). These bifurcations have been fully characterised analytically for strings of cells (a) where $K=\cos (2 \pi / \omega)$, for integer wavelengths $\omega$, and similar trends are observed for squares (b) and hexagons (c). We use the functions $K$ from the homogeneous cell case (Webb and Owen, 2003) as convenient mappings for squares and hexagons: $K=\left(\cos \left(2 \pi / \omega_{1}\right)+\cos \left(2 \pi / \omega_{2}\right)\right) / 2$ for squares, and $K=\left(\cos \left(2 \pi / \omega_{1}\right)+\cos \left(2 \pi / \omega_{2}\right)+\cos \left(2 \pi / \omega_{1}+2 \pi / \omega_{2}\right)\right) / 3$ for hexagons, where $\omega_{1}$ and $\omega_{2}$ are the integer wavelengths for the horizontal and vertical directions, respectively. Similar trends in pattern wavelengths and bifurcations are predicted for this model and the case of cellular homogeneity (see Figure 1 for a comparison). Wavelengths (and $K$ ) increase from left (black) to right, and longer wavelengths are more likely for weak lateral inhibition or lateral induction. The pattern regions for squares/hexagons are qualitatively similar to strings, except that the equivalent curve to $T_{d}$ cannot be easily characterised (e.g. see zoom box in (b)). The pattern region is smaller for hexagons, so that weak or negative receptor upregulation cannot support patterns (for more detail see Figure 8). The parameter values are as in Appendix D, except that $D_{a}=0.1, D_{f}=0$, and there is unbiased global production $\left(\gamma_{a}, \gamma_{f}=0\right)$. 
(a) Stationary

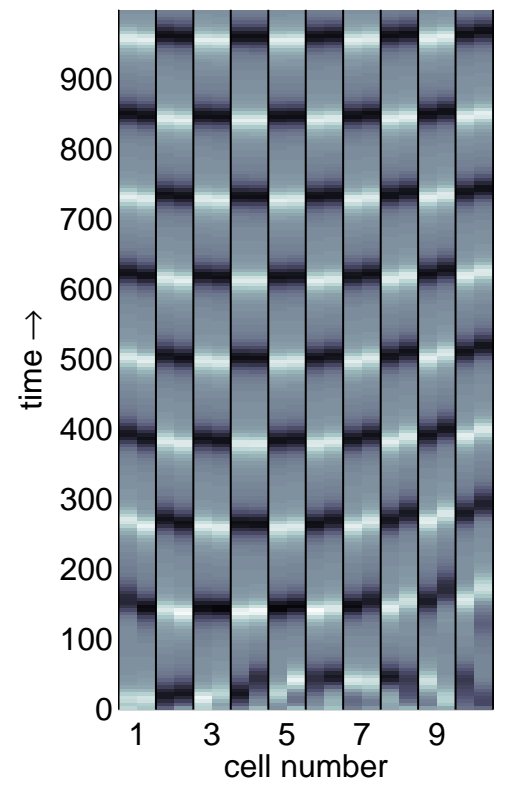

(b) Travelling

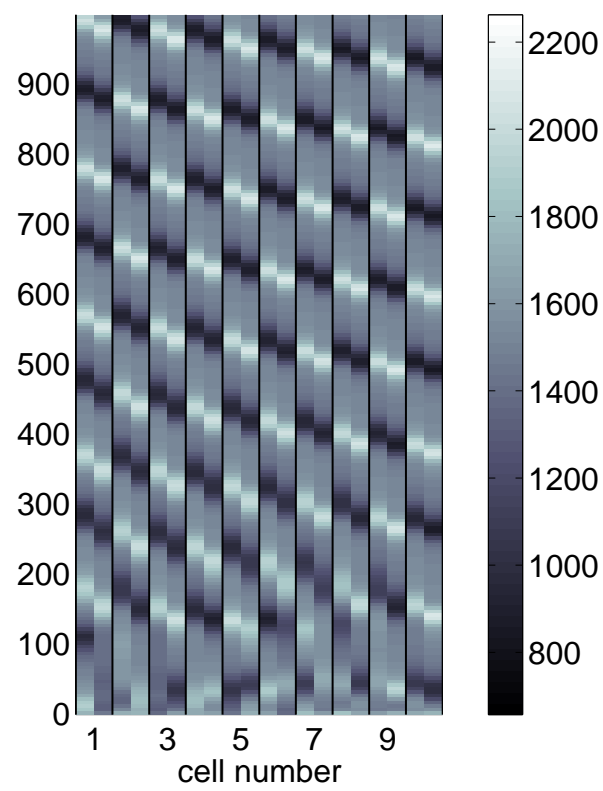

Figure 6: Standing and travelling waves in the juxtacrine model (1) with unbiased global production $\left(\gamma_{u}=0\right)$ on a string of 30 cells with periodic boundary conditions - for clarity, we show a subset of 10 cells. We plot the densities of bound receptors at successive times - ligand/free receptor profiles are similar. The initial conditions are small random perturbations about the homogeneous equilibrium. The ligand and receptor production parameters are $m=-4.3$ and $n=-0.5$, giving $\mathcal{A}=-0.431, \mathcal{F}=-0.048$. The other parameters are the same as in Appendix D, except that $k_{i}=0.19 \mathrm{~min}^{-1}, D_{a}, D_{f}=0$ and $\gamma_{a}, \gamma_{f}=0$. 
(a)

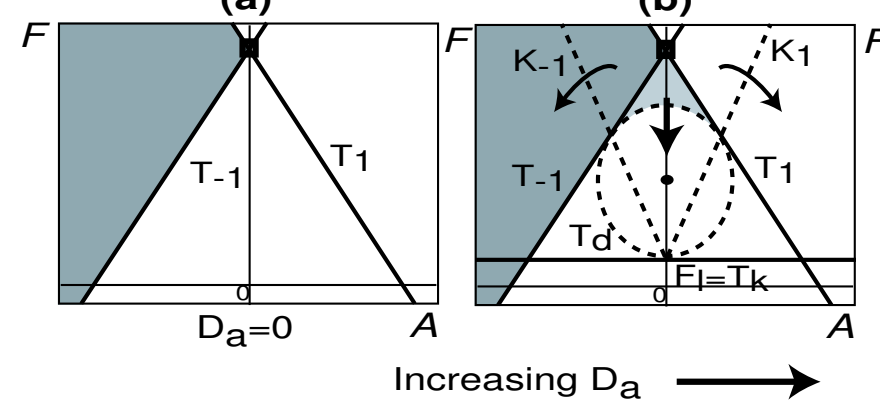

(d)

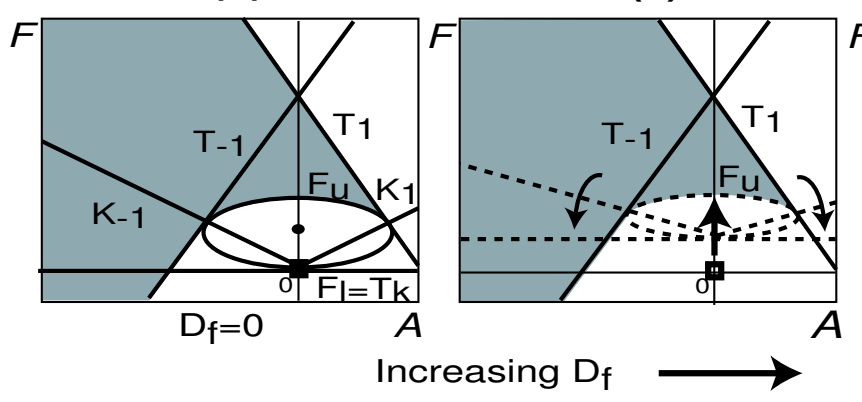

(c)

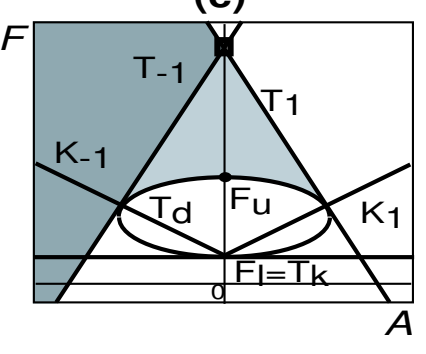

(f)

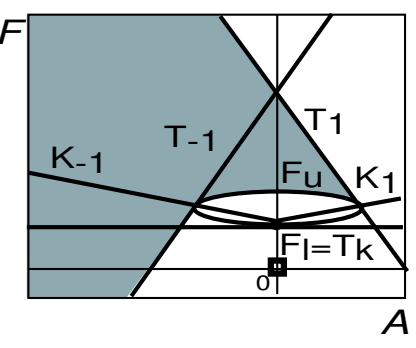

Figure 7: Qualitative illustration of the modulation of patterning by intra-membrane diffusion, $D_{a}$ and $D_{f}$. Patterning instabilities are predicted in the shaded regions. The upper (lower) row shows the effect of increasing $D_{a}\left(D_{f}\right)$, with $D_{f}\left(D_{a}\right)$ fixed - the arrows indicate the direction of increasing $D_{a}, D_{f}$. With no intra-membrane ligand diffusion, $D_{a}=0$ (part (a)), the ellipse $T_{d}$ is not defined, the pattern region is smaller, and in particular there is no pattern formation for lateral induction $(\mathcal{A}>0)$. Increasing $D_{a}$, makes the ellipse $T_{d}$ more horizontal, and increases the size of the patterning region (parts (b) and (c)). Increasing $D_{f}$ has less effect (parts (d)-(f)). 


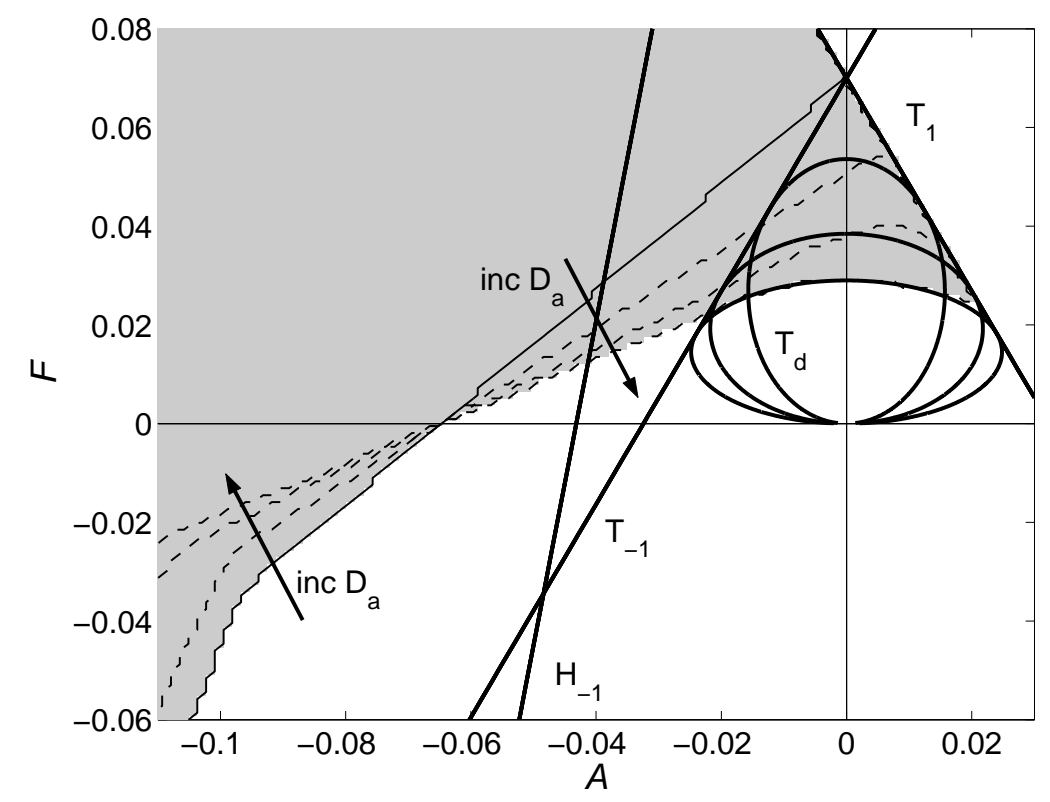

Figure 8: Intra-membrane ligand diffusion, $D_{a}$, modulates the patterning region (shaded) for hexagonal arrays. The thick solid diagonal lines and ellipses are the analytically derived bifurcations for strings, and the thin lines indicate the bifurcations for hexagons (solid line is when $D_{a}=0$, dotted lines are for $D_{a}=0.01,0.05$ and 100). Increasing $D_{a}$ enlarges the size of the pattern region for lateral induction $(\mathcal{A}>0)$, but makes patterns less likely for lateral inhibition when $\mathcal{F}<0$. The parameter values are as in Appendix D, except $D_{f}=\gamma_{a}=\gamma_{f}=0$. 
(a)

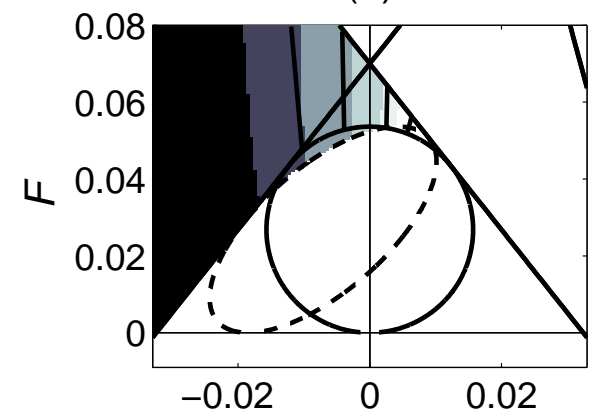

(c)

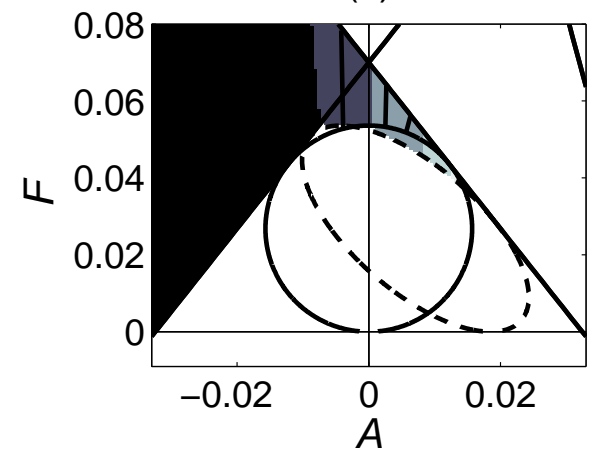

(b)

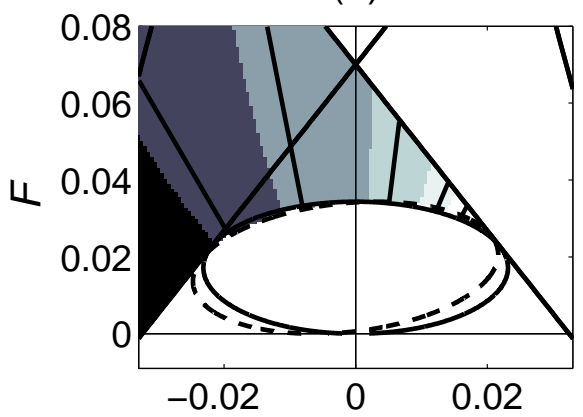

(d)

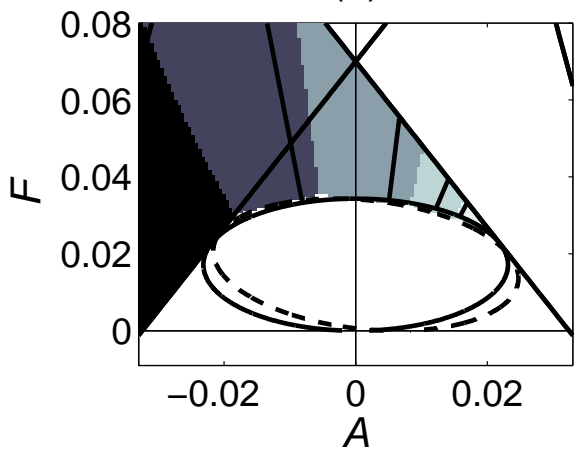

Figure 9: Intra-membrane ligand diffusion, $D_{a}$, and biased ligand production, $\gamma_{a}$, modulates patterning in strings of cells. Wavelengths increase from left to right (black is for wavelength two, light grey is for longer wavelengths). In unshaded regions there is no patterning instability. The solid diagonal lines and ellipses are analytically derived bifurcations $\left(T_{ \pm 1}, H_{ \pm 1}\right.$ and $\left.T_{d}\right)$, and the solid lines emanating from the ellipses are transitions of the fastest growing mode when $\gamma_{a}=0$. The dashed ellipses show the bifurcations when $\gamma_{a}= \pm 1$, and the shadings indicate the transitions between fastest growing modes. In (a) $\gamma_{a}=-1, D_{a}=0.01$; (b) $\gamma_{a}=-1, D_{a}=0.1$; (c) $\gamma_{a}=1, D_{a}=0.1$; (d) $\gamma_{a}=1, D_{a}=0.1$. The other parameters are given in Appendix D. Increasing $D_{a}$ enlarges the patterning region and makes longer wavelengths more likely (compare left and right column). Altering the bias in ligand production has less effect on the size of the pattern region but modulates the length of the predicted wavelengths (compare upper and lower rows). 

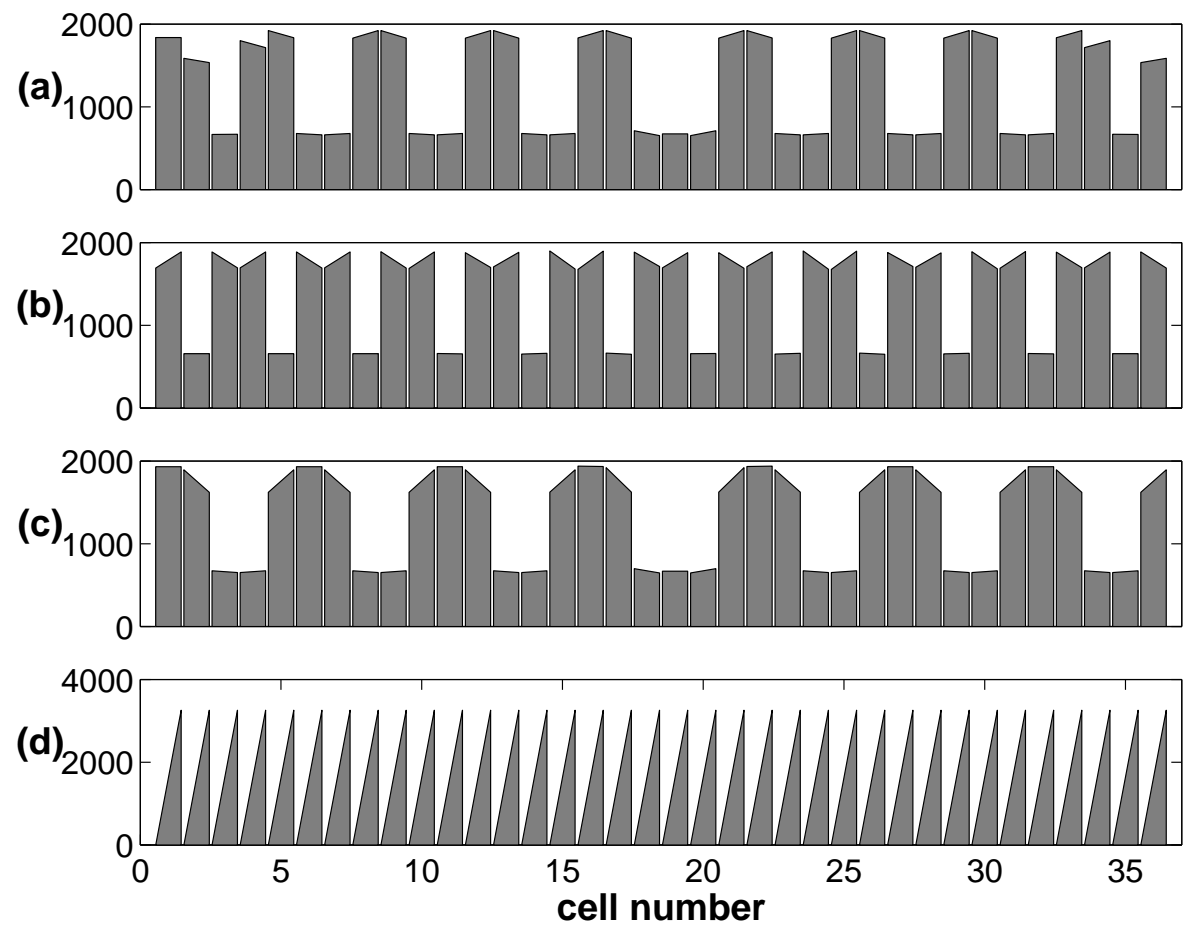

Figure 10: Numerical simulations of the juxtacrine model (1), solved on a string of 36 cells with different combinations of biased ligand and receptor production. In $(\mathrm{a}-\mathrm{c})$ the strengths of production are $m=-0.1, n=7$ (giving $\mathcal{A}=-0.0012, \mathcal{F}=0.0665$ ) while $\gamma_{a}, \gamma_{f}$ are allowed to vary. We show the numbers of bound receptors on both sides of each cell - profiles for ligand and free receptor are similar. From top to bottom: (a) unbiased global production $\left(\gamma_{a}=\gamma_{f}=0\right)$ still gives differences between sides due to ligand-receptor binding; (b) $\gamma_{a}=\gamma_{f}=1$ enhances differences and the wavelength shortens; (c) $\gamma_{a}=\gamma_{f}=$ -1 gives a homogenising effect and increases the predicted wavelength. (d) Shows a regular pattern of polarity across the array: $\gamma_{a}=-1, \gamma_{f}=1$, and $m=-0.6, n=2.5$ (giving $\mathcal{A}=-0.0071, \mathcal{F}=0.0238$, within the region of stability in $(\mathcal{A}, \mathcal{F})$ space). The other parameters are the same as in Appendix D, with $D_{a}=0.01, D_{f}=0.01$ in $(\mathrm{a}-\mathrm{c})$ and $D_{a}=0, D_{f}=0$ in $(\mathrm{d})$. The initial conditions are given by the homogeneous equilibrium, except for a small perturbation of the left-hand side of the first cell. 

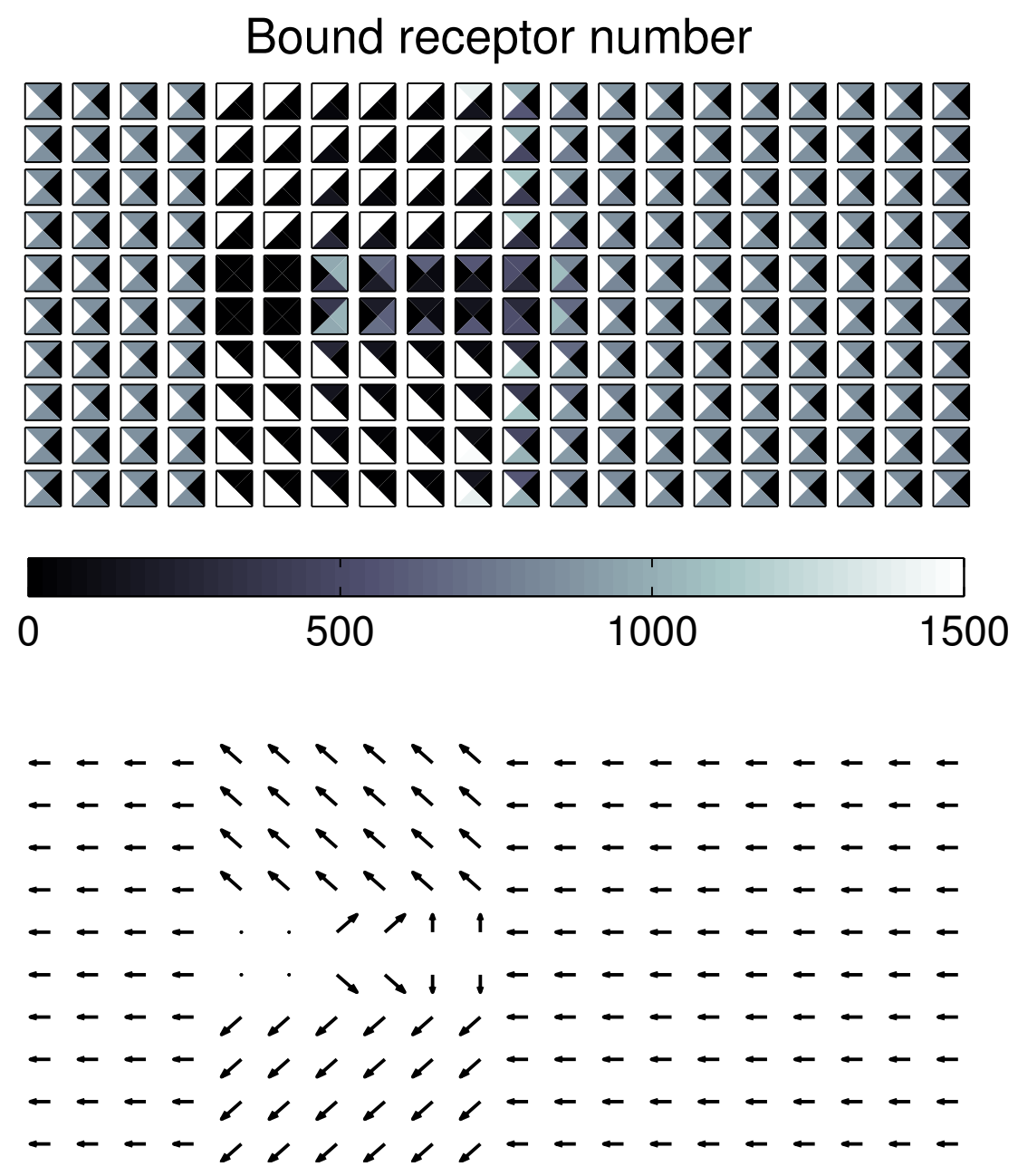

Figure 11: Numerical simulations of the juxtacrine model (1), solved on a $10 \times 20$ array of square cells. We explore how defects in signalling in a small group of cells can propagate into the surrounding region of regularly polarised cells. In the upper row we show the number of bound receptors - ligand and free receptor profiles are similar. The arrows in the bottom row point in the direction of the side with the highest bound receptor number. The parameters are given in Appendix D, except that $m=1, n=4$ (giving $\mathcal{A}=$ $0.012, \mathcal{F}=0.038)$, and $D_{a}, D_{f}, \gamma_{a}, \gamma_{f}$ are all taken to be zero. The boundary conditions are zero flux. 

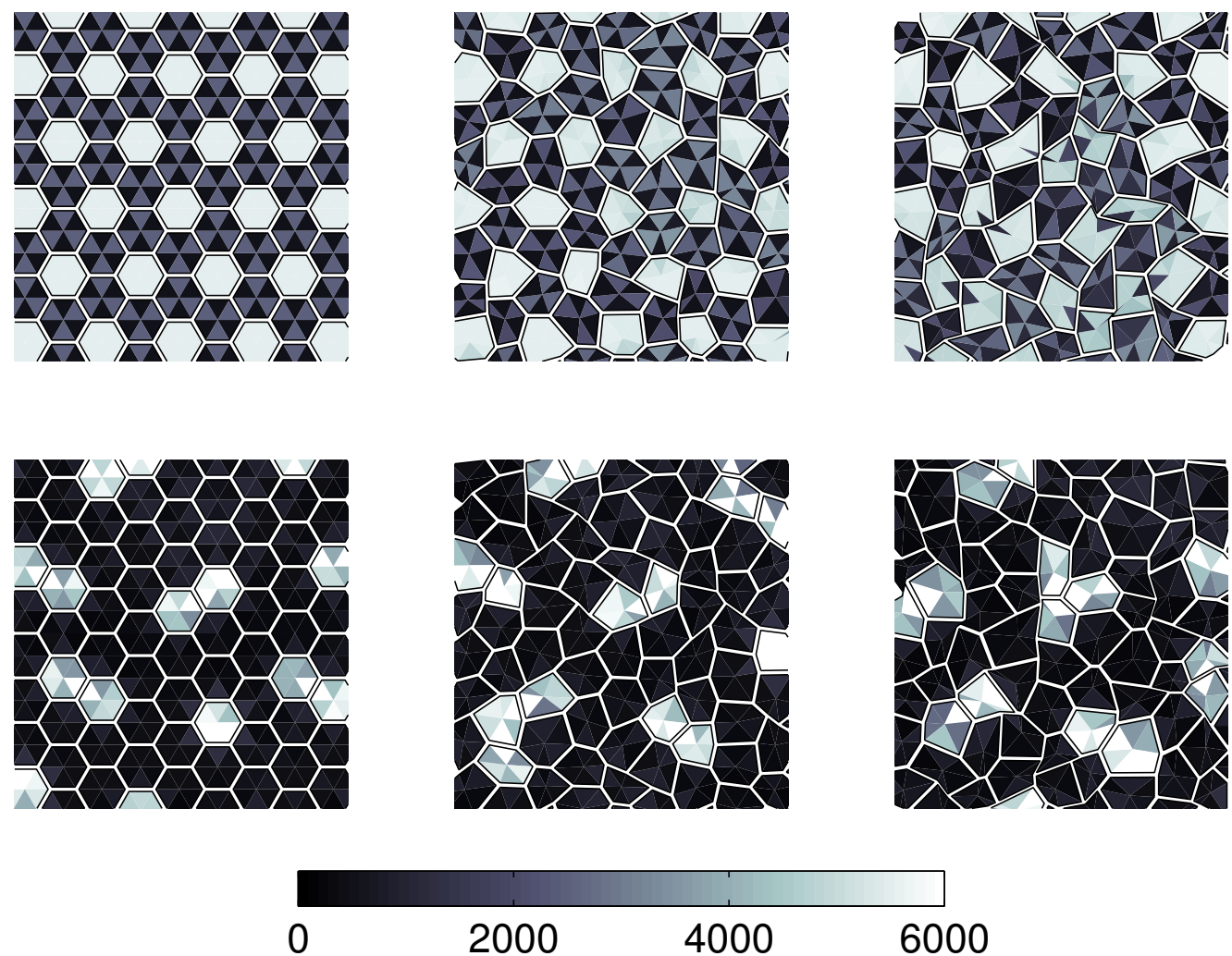

Figure 12: Numerical simulations of the juxtacrine model (1) on hexagonal arrays with varying random perturbations of a regular structure (first column). Small perturbations (second column) still support regular patterns, but larger perturbations (third column) lead to some irregularities. Similar results are seen with both lateral inhibition and lateral induction (upper and lower rows respectively). Regular hexagons of side 1 unit are generated and the co-ordinates of their vertices are perturbed by a random number drawn from a uniform distribution on $[0,0.7]$ (second column), or [0,1] (third column). The parameter values are as in Appendix D except: (upper row) $m=-4, n=2$ and $D_{a}=0.0014 ;$ (lower row) $m=2, n=3, d_{a}=0.01, d_{f}=0.03, r_{0}=900, \beta_{a}=0.2, \beta_{f}=1.5$ and $D_{a}=0.1$. The diffusive terms are as defined in Appendix E. 
Time $=0$

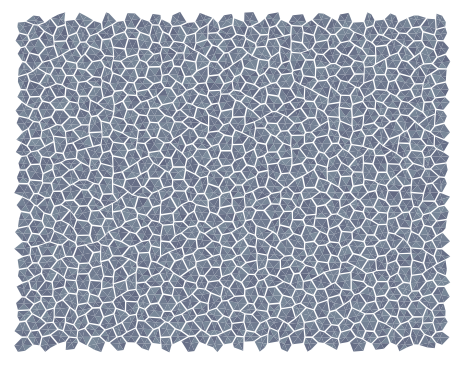

Time $=0$

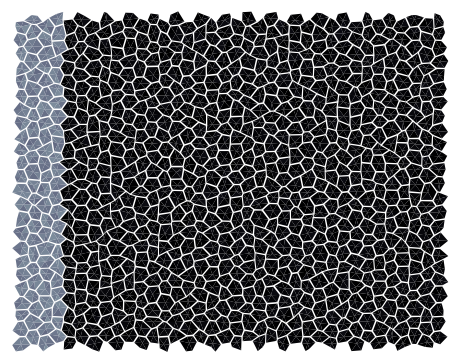

Time $=0$

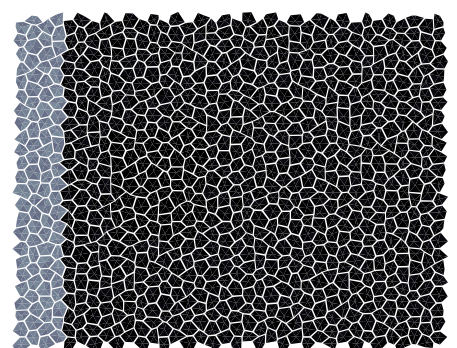

Time $=500$

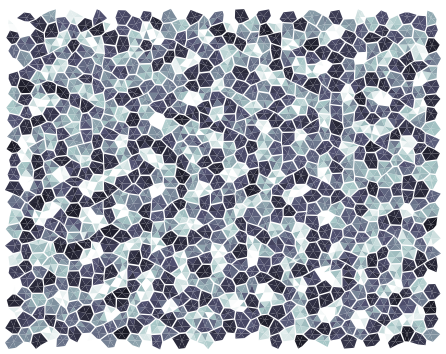

Time $=1500$

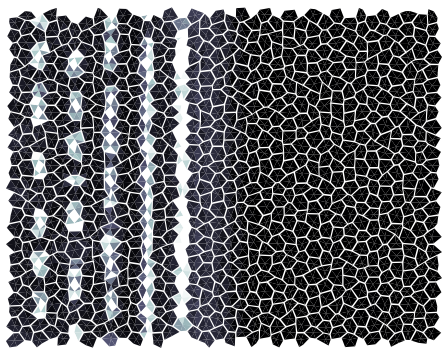

Time $=1500$

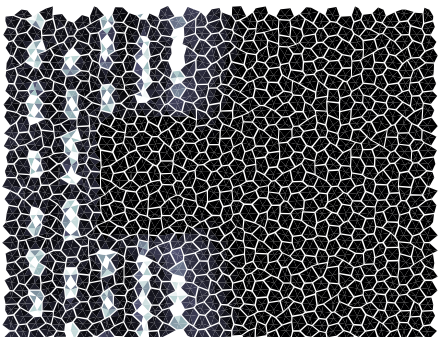

Time $=3000$

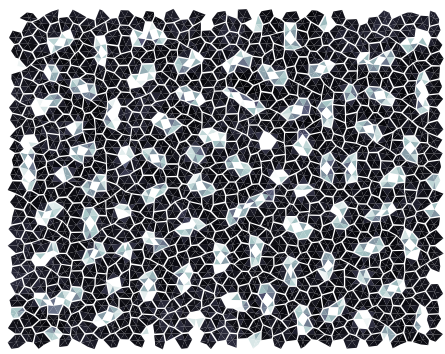

Time $=3000$

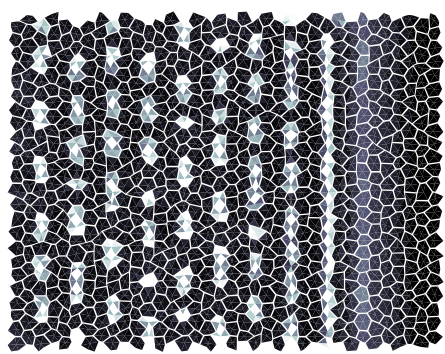

Time $=3000$

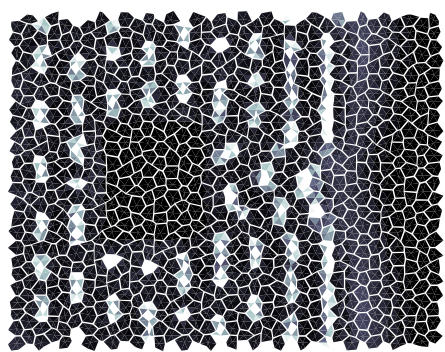

6000

Figure 13: Travelling waves in the extended L-R model (1) with inhomogeneous protein distributions, and irregular geometries as outlined in Appendix E. The upper row shows the evolution of an irregular pattern generated from random initial conditions of the active homogeneous steady state. Pattern initiation by wave activation can generate more regular patterns behind the advancing wave front (second row). The third row illustrates how relay induced patterning is undisturbed by clones of non-signalling cells - contrary to current biological thinking (Strutt, 2002). The parameter values are the same as for the lower row of Figure 12. 